\title{
Autour du Mur (dit) d'Hannibal : appréhender un "dispositif militaire » du premier siècle av. J.-C. dans les Alpes poenines (Valais, Suisse et Vallée d'Aoste, Italie)
}

\author{
Romain Andenmatten ${ }^{1}$
}

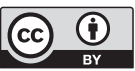

Reçu : 29/07/2020

Accepté : 05/12/2020

\section{Résumé}

Des sites occupés par des militaires romains durant la seconde moitié du premier siècle avant J.-C. ont été mis en évidence entre 2300 et $3100 \mathrm{~m}$ d'altitude dans les Alpes poenines. Après une introduction au programme de recherche sur ces positions, un catalogue présente les résultats des investigations sur les sites valaisans. La confrontation de ces découvertes aux sources historiques et plus particulièrement aux événements ayant amené à l'établissement de l'hégémonie romaine sur cette région, ainsi que le rappel des réserves méthodologiques inhérentes à cet exercice font office de conclusion. L'ensemble amène un nouvel éclairage sur la période tardo-républicaine dans cette partie des Alpes et présente un état des lieux de cette recherche toujours en cours.

Mots-clés : archéologie alpine ; haute montagne ; époque tardo-républicaine ; hégémonie romaine ; armée romaine ; dispositif militaire

Abstract. Around the Hannibal's Wall: apprehending a "tactical mapping" of the first century BC in the Poenines Alps (Valais, Switzerland and Aosta Valley, Italy)

Sites occupied by Roman soldiers during the second half of first century BC have been found between 2300 and $3100 \mathrm{~m}$ altitude in the Poenine Alps. After an introduction to the research program on these positions, a catalog presents the results of the investigations on the Valais sites. The confrontation of these discoveries with historical sources and, more particularly, with the events that led to the establishment of Roman hegemony over this region, as well as the reminder of the methodological reservations inherent to this exercise, serve as a conclusion. All this cast a new light over the late-republican period in this part of the Alps and presents a state of this ongoing research.

Keywords: alpine archaeology; high mountain; late Roman Republic; Roman hegemony; Roman army; tactical mapping

1. Groupe scientifique RAMHA, Association RAMHA, Maison de commune, 1945, Liddes. romain.andenmatten@admin.vs.ch Avec les contributions de Paul-Emile Mottiez ( Illustration des objets et détermination des monnaies ) et Aurèle Pignolet ( Plans des sites ). Tous nos remerciements vont à Julien Andenmatten pour sa relecture critique et attentive. 
Andenmatten, Romain. «Autour du Mur (dit) d'Hannibal : appréhender un « dispositif militaire " du premier siècle av. J.-C. dans les Alpes poenines (Valais, Suisse et Vallée d'Aoste, Italie)». Treballs d'Arqueologia, 2020, núm. 24, p. 133-164. DOI: 10.5565/rev/tda.124

\section{Introduction}

\subsection{Pourquoi un article sur un projet d'étude dans les Alpes lors des actes d'un colloque pyrénéen?}

Un peu d'exotisme mais pas seulement!

Nous avons rencontré Oriol Olesti

Vila pendant une session sur l'approche comparative de la conquête romaine durant un colloque à Édimbourg en 2018. Le contact fut immédiatement amical et malgré la distance géographique séparant nos milieux d'étude, leurs similitudes ainsi que nos centres d'intérêts communs nous ont rapidement rapprochés et amenés à échanger sur nos travaux en cours. C'est ainsi que des archéologues travaillant sur le milieu alpin et plus particulièrement sur les modalités de la transition entre l'âge du Fer et l'époque romaine dans les Alpes poenines se sont retrouvés à la Cerdagne pour présenter les résultats préliminaires de leurs recherches dans le cadre d'ARQUEOPYRENAE-2.

Le programme d'investigation mené ces quinze dernières années en haute altitude entre le Valais et la Vallée d'Aoste a permis de mettre en évidence un ensemble insoupçonné de sites qui permettent aujourd'hui de réécrire une partie de l'histoire de la mise en place de l'hégémonie romaine dans les Alpes poenines. Cette étude n'est de loin pas terminée et les recherches planifiées ces prochaines années permettront d'affiner ou de corriger les hypothèses proposées.

\subsection{Un mur, une inscription, un projet de recherche...}

Les recherches interdisciplinaires autour du Mur (dit) d'Hannibal se sont tout d'abord concentrées sur leur site éponyme dans la commune de Liddes en haut Val d'Entremont (Région du Grand-SaintBernard, Valais, Suisse). Cet endroit, associé à plusieurs légendes locales, interpellait depuis des décennies les érudits de la région ou de passage mais n'avait jamais été le lieu d'une découverte et encore moins l'objet d'une étude archéologique. ${ }^{2}$ En août 2005, l'invention d'une inscription en alphabet (dit) de Lugano allait relancer l'intérêt autour du site et peut probablement être considérée comme l'un des principaux éléments déclencheurs de nos recherches. ${ }^{3}$ Les premières investigations de terrain (de 2006 à 2010) ont été le fait d'étudiants bénévoles de l'Université de Lausanne encadrés par un archéologue chargé d'un mandat d'exploration du Canton du Valais. ${ }^{4}$ En 2011, un travail

2. Andenmatten et Paccolat $(2010: 311-313)$; Andenmatten et Paccolat $(2012: 79)$.

3. Quartier-La-Tente (2007: 12-13) ; Andenmatten et Paccolat (2012: 79-80 et 91-92) ; Casini et al. (2013: 157) ; Aberson et al. (2021 : 309-332).

4. Travaux conduits par Romain Andenmatten sous la responsabilité scientifique d'Olivier Paccolat; Andenmatten (2011); Andenmatten et Paccolat (2012). 
de mémoire de maîtrise à l'Université de Lausanne et la fondation d'une association de soutien aux recherches archéologiques sur le Mur (dit) d'Hannibal (RAMHA) ont constitué les bases permettant la conduite de trois nouvelles campagnes (2014 à 2016) sur les hauts de Liddes par un groupe scientifique interdisciplinaire, puis de trois campagnes ponctuelles sur six positions valaisannes présentant des caractéristiques proches $\left(2016,2018\right.$ et 2019). ${ }^{5}$

\subsection{Recherches en Vallée d'Aoste et étude transnationale}

En Vallée d'Aoste (Italie), le site du Mont Tantané, dans la commune de La Magdeleine, avait déjà été repéré en 1970. C'est pourtant plus de 30 ans plus tard qu'il fait l'objet d'investigations de grande envergure (2003-2005, 2009-2010). L'étude des résultats des fouilles est actuellement en cours. ${ }^{6}$

En parallèle, de nouveaux sites sont repérés par des membres de la Société valdôtaine de préhistoire au milieu des années 2000 puis par un groupe de randonneurs passionnés du passé et des cimes de la région. Une dizaine de nouvelles positions sont ainsi recensées jusqu'en 2018. ${ }^{7}$
Les premiers contacts pour la création d'un groupe de travail transnational sont établis en 2017 entre l'équipe RAMHA et les responsables de la surintendance valdôtaine, puis dès 2018 débutent des recherches en commun sur l'arête frontière entre le Valais et la Vallée d'Aoste. Ces dernières se poursuivent en 2019. En 2020, le projet réunit ainsi un groupe d'une vingtaine de chercheurs issus des sciences environnementales et humaines rattachés à des services publics et à des organismes de recherche ou professionnels indépendants. Près d'une vingtaine de sites de haute altitude (entre 2300 et $3100 \mathrm{~m}$ ) présentant des caractéristiques similaires au Mur (dit) d'Hannibal sont recensés et un programme d'étude est planifié pour les appréhender dans les années à venir. ${ }^{8}$ Un site similaire aurait également été repéré en Tarentaise (France), à une vingtaine de kilomètres au sud-ouest du Petit-Saint-Bernard et pourrait, à moyen terme, permettre d'élargir la zone d'intérêt du programme d'étude en cours. ${ }^{9}$

\subsection{Conditions et méthode}

En 2020, le projet est conduit dans le cadre d'un partenariat entre l'association

5. Andenmatten et Pignolet (2015a : 525-527) ; Andenmatten et Pignolet (2015b : 297-299) ; Andenmatten et Pignolet (2016a : 313-316) ; Andenmatten et Pignolet (2016b : 226-233 et 248) ; Andenmatten et Pignolet (2017: 41-44); Andenmatten et al. (2020, à paraître).

6. Armirotti (2003: 140-141) ; Mezzena (2004: 157) ; Bertocco (2017 : 87 et 90) ; Gwenaël Bertocco étudie la céramique provenant du Mont Tantané dans le cadre de sa thèse et le groupe de travail commun entre la Surintendance pour les activités et les biens culturels de la Région autonome Vallée d'Aoste et le groupe scientifique RAMHA a entrepris en 2019 de poursuivre l'étude de ce site : élaboration de la documentation et étude de l'ensemble du mobilier.

7. Daudry (2005); Reboulaz (2005). Consultation le 28 mai 2020 :

$<$ https://andarpersassi.it/antichi-insediamenti-in-quota/\#content>;

$<$ https://andarpersassi.it/villaggi-salassi-aggiornamento/\#content>;

$<$ https://andarpersassi.it/villaggi-salassi-nuovo/\#content>.

8. Andenmatten et Aberson (2019: 219-221).

9. Information orale de Pierre-Jérôme Rey, un collègue qui devrait publier prochainement une notice sur le sujet. 
Autour du Mur (dit) d'Hannibal : appréhender un "dispositif militaire » du premier siècle av. J.-C. dans les

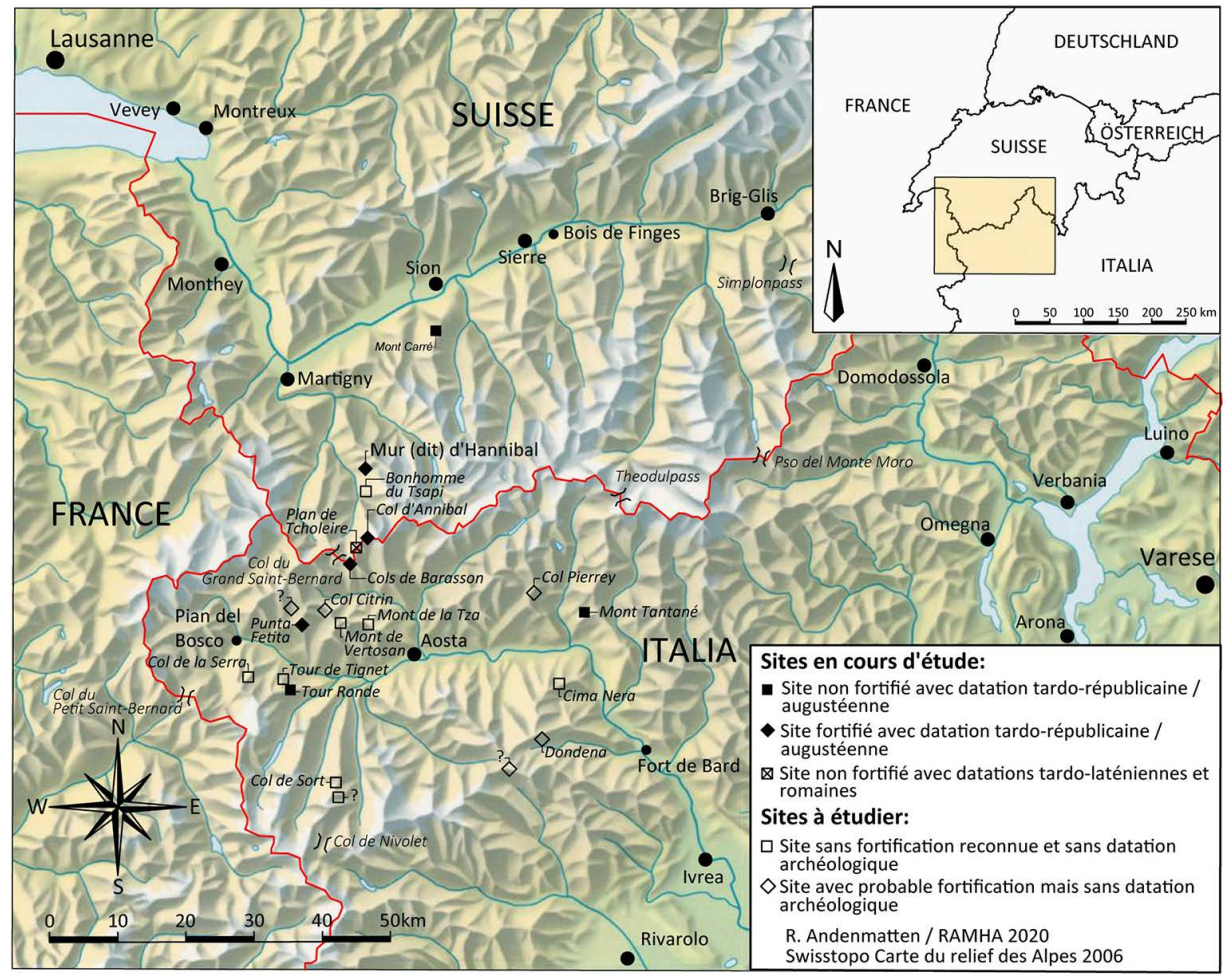

Figure 1. Carte de situation des sites en cours d'étude et à étudier (C RAMHA 2020).

RAMHA et l'Office cantonal d'Archéologie en Valais ainsi que dans le cadre d'une collaboration entre le groupe scientifique RAMHA et la Surintendance pour les activités et les biens culturels en Vallée d'Aoste. ${ }^{10}$

Le projet intègre les spécialistes associés dès les premières étapes d'investiga- tions de terrain sur les sites et bénéficie ainsi de leurs conseils dans la conduite des opérations archéologiques. ${ }^{11}$ Des tables rondes générales ou thématiques sont également organisées pour permettre aux chercheurs de développer leurs travaux en conservant une vue d'ensemble de l'avancement des études des autres.

10. L'association RAMHA est représentée par son Président, Monsieur Jean-François Copt et son responsable du groupe scientifique, Monsieur Romain Andenmatten, l'Office cantonale d'Archéologie par Madame Caroline Brunetti, archéologue cantonale et la Surintendance pour les activités et les biens culturels par Madame Alessandra Armirotti, instructrice technique et responsable des questions liées à l'époque romaine.

11. Le groupe de travail comprend des archéologues, géologues, glaciologues, palynologues, archéozoologues, archéobotanistes, anthracologues, historiens, épigraphistes, linguistes, spécialistes en sciences forensiques, typologues, dendrochronologues, etc. 
Pour parfois valider ou infirmer des résultats, plusieurs spécialistes sont aussi amenés à travailler chacun en vase clos avant de se confronter. Une critique croisée, ouverte et constructive des travaux de chacun est également encouragée en permanence. Des spécialistes extérieurs au projet sont enfin appelés à participer aux discussions pour y apporter leurs regards critiques.

Les sites archéologiques de haute altitude présentent plusieurs contraintes mais également des avantages que notre groupe de recherche tente d'exploiter au mieux. Parmi les contraintes, l'une des premières est d'ordre éthique car les interventions sont exclusivement réalisées sur des sites sans danger imminent. Les investigations invasives et de fait destructives ont ainsi été réduites au minimum en se limitant à une fouille partielle des bâtiments ${ }^{12}$, en privilégiant la réalisation de sondages ponctuels de petites dimensions (souvent d'un quart de mètre carré, voire moins) et en utilisant le détecteur de métaux avec une méthodologie stricte (secteurs de prospection clairement définis, géoréférencement millimétrique des découvertes, dessins des profils stratigraphiques de référence pour l'insertion des objets typologiques, documentation des niveaux d'occupation et prélèvement des sédiments d'occupation pour analyses). Les sites explorés n'ont ainsi pas vocation à devenir des chantiers d'investigation dans la longue durée mais d'être conservés tels des réserves archéologiques après la réalisation d'une à quelques campagnes de recherches à l'impact limité.

Une autre caractéristique contraignante à laquelle nous sommes confrontés n'est autre que la réoccupation des mêmes emplacements tandis que les stratigraphies y sont caractérisées par une érosion forte et des dépôts sédimentaires faibles à inexistants. Les fouilles ne permettent ainsi que très rarement l'établissement d'une chronologie relative des structures et ce même pour des secteurs étudiés sur des surfaces d'une à quelques dizaines de mètres carrés. Trois foyers documentés dans un sondage fouillé en 2015 sur le site du Mur (dit) d'Hannibal (14 $\mathrm{m}$ de longueur par $1 \mathrm{~m}$ de largeur) présentaient ainsi une insertion stratigraphique similaire : au-dessus d'un paléosol piétiné à la surface du substrat périglaciaire et en partie comprise dans l'humus actuel avec des charbons affleurant presque en surface. ${ }^{13}$ Leurs datations par le radiocarbone au mésolithique, entre les premiers siècles avant et après notre ère ainsi que postérieure au milieu du $20^{\mathrm{e}}$ siècle illustrent cependant la diachronie d'occupation de la position et appellent à la prudence quant à l'interprétation des structures dont la datation est incertaine en haute montagne. ${ }^{14}$

12. Seul le premier bâtiment investigué a été exploré sur l'entier de sa surface sur le site du Mur (dit) d'Hannibal et les bâtiments suivants ont été explorés au maximum sur leur moitié.

13. La lecture de la végétation s'est avérée utile dans le choix des emplacements des sondages, car à une altitude où les sols sont très pauvres et l'humus peu épais (parfois $<5 \mathrm{~cm}$ ) la présence de foyers modifie les ressources à la disposition des plantes. Ainsi certains types de fleurs semblent " anthracophiles » et pourraient servir d'indicateurs de la présence de structures de combustion. Une étude serait à développer sur cette question.

14. Mur (dit) d'Hannibal : foyer US148, BE-11632.1.1, charbon de bois (pinus), 7340 (92.9\%) 7137 calBC / $7102(2.5 \%) 7085$ calBC et BE-11926.1.1, charbon de bois (pinus), 7330 (92.6\%) 7137 calBC / 7101 (2.8\%) 7085calBC; foyer US150, BE-11637.1.1, macroreste (hordeum), 86 (1.2\%) 78calBC / 
Plusieurs positions ont également fait l'objet de perturbations importantes ou ont été irrémédiablement endommagées au siècle dernier. Il en est ainsi du Mur (dit) d'Hannibal qui se trouva au centre d'une zone des buts pour l'artillerie pendant plusieurs décennies du $20^{\mathrm{e}}$ et jusqu'au début du $21^{\mathrm{e}}$ siècle. ${ }^{15}$ Les impacts, tout comme les éclats d'obus, y sont très nombreux. Tout en ayant endommagé la position, on peut cependant aussi considérer que la grande quantité de métaux divers épandus sur la position constitue une protection contre le pillage par des pratiquants de la détection illicite. Autre exemple, le Col Ouest de Barasson a, comme les autres positions de l'arête frontière, été occupé pendant la mobilisation du second conflit mondial. Cependant ces aménagements n'ont qu'un impact limité (réutilisation de pierres pour la construction d'une à deux cabanes). Par contre, ce site a été choisi pour le passage de deux lignes à haute tension (dans les années 1950-1970) et les restes laissés lors des travaux de construction des pylônes ont largement pollué le secteur tandis que l'installation de mises à terre (sur parfois plus de $50 \mathrm{~m}$ de longueur) a également labouré le sous-sol du col.

Les passages réguliers de chasseurs, de randonneurs ou d'alpinistes, dont certains ont réoccupé des abris anciens par économie de moyens n'ont eu que des impacts très ponctuels sur la conservation des vestiges des positions explorées.
Il faut aussi relever que dans les cas où les aménagements se situent à la surface de moraines anciennes et totalement lessivées de leurs matrices fines à moyennes ou dans des éboulis (par exemple, le secteur des abris situés dans les moraines au nord-ouest de l'enceinte principale du Mur (dit) d'Hannibal et la totalité du site du Col d'Annibal), aucun niveau archéologique et aucun petit mobilier ne sont généralement conservés.

On mentionnera en revanche l'avantage de la conservation particulièrement bonne du mobilier métallique enfoui, principalement du fer découvert presque sans gangue de corrosion. ${ }^{16} \mathrm{La}$ conservation occasionnelle d'éléments en matériaux périssables remontant à l'Antiquité (des fragments de bois à la surface et à la périphérie de zones englacées mais également dans des niveaux d'occupation) est aussi l'une des caractéristiques exceptionnelles de l'archéologie de haute montagne. Ces conservations rendent possibles des études parfois bien plus ardues, voire impossibles, sur d'autres sites terrestres (dendrologie, dendrochronologie, étude typologique d'objets en matériaux mous : bois, cuir, textiles, etc.).

Le fait qu'à ces altitudes, les occupations de grande ampleur soient nécessairement saisonnières et le plus couramment événementielles permet aussi d'appréhender les niveaux d'habitat compris dans leurs bâtiments comme des ensembles presque clos.

55calBC (91.6\%) 30calAD / 38 (2.5\%) 50calAD ; foyer US146, BE-11927.1.1, charbon de bois (Alnus), Bomb Carbon Effect / post 1955 AD ; OxCal v4.3.2 (Bronk Ramsey, 2017), r5 , IntCal13 atmospheric curve (Reimer et al., 2013).

15. Les tirs d'artillerie sur le Mur (dit) d'Hannibal se sont poursuivis jusqu'au milieu des années 2000 et ont été stoppés à l'instigation de Monsieur Vincent Quartier-La-Tente.

16. Pour les objets enterrés, cet état de conservation n'est cependant que de courte durée car, dès leur sortie de leur milieu d'enfouissement, ils présentent rapidement des symptômes de réactivité avec un nouveau processus de corrosion (voir Scholl, $2015: 27-32$ ). 


\section{Un catalogue de sites}

Les sept sites repérés en Valais à ce jour font ici l'objet d'une courte description. L'état des connaissances est développé pour chaque position en parallèle de l'ampleur des investigations conduites. Les éventuels liens entre les positions, leur probable hiérarchie ainsi que les interprétations qui en découlent sont abordés à la suite du catalogue. Les sites valdôtains ne sont pas présentés en détail et feront l'objet d'un article commun avec nos confrères de la Surintendance pour les activités et les biens culturels.

Pour chaque site, une planche présente une sélection du mobilier typologique correspondant principalement à la période de transition entre l'âge du Fer et l'époque romaine tandis qu'un plan simplifié permet de donner une idée de la situation des principaux aménagements.

\subsection{Le Mur (dit) d'Hannibal (Liddes, Valais, Suisse, $2650 \mathrm{~m}$ )}

Le nom Mur (dit) d'Hannibal est issu de la tradition orale locale et n'a été mentionné pour la première fois par écrit qu'en $1983 .{ }^{17}$ Des ruines avaient cependant déjà été observées à l'emplacement du site lors de l'élaboration du premier atlas national en $1878 .{ }^{18}$

Il s'agit de la position de notre corpus dont l'étude est la plus avancée. Des recherches de terrain y ont été conduites entre 2006 et 2019 pour une période cu- mulée de cinq mois avec une équipe de 5 personnes en moyenne. Des prospections ciblées, des petits sondages mais également des fouilles sur quelques dizaines de mètres carrés ont pu être réalisées dans le cadre de ces investigations. ${ }^{19}$ L'étude de ce site sert de référence dans l'élaboration des réflexions sur les autres positions.

Le Mur (dit) d'Hannibal est situé à env. $2650 \mathrm{~m}$ d'altitude, sur un contrefort du flanc oriental du Val d'Entremont. L'enceinte principale, de $270 \mathrm{~m}$ de longueur et jusqu'à $2.1 \mathrm{~m}$ de hauteur, est appuyée contre un à-pic et délimite une zone protégée d'env. $3500 \mathrm{~m}^{2}$. Un second mur relie la forteresse à un promontoire situé à une centaine de mètres au sud et barre ainsi l'unique voie d'accès aisée à la position depuis le sud.

Un total de 40 fonds de cabanes vérifiés et 10 fonds de cabanes probables ont été repérés ainsi que 37 abris aménagés sous ou contre des blocs et 6 probables abris similaires. Parmi les structures, le petit abri dans lequel est conservée l'inscription en alphabet (dit) de Lugano mérite d'être mis en évidence. ${ }^{20}$

L'ensemble des datations effectuées dans les bâtiments ainsi que les analyses micromorphologiques réalisées amènent à considérer l'occupation principale du site comme événementielle sur une à quelques saisons (probablement pas plus de 3-4 saisons successives) dans une fourchette large située dans les deux derniers tiers du premier siècle avant J.-C. Les datations des niveaux d'occupation de certains bâtiments ainsi que de structures

17. Lattion (1983).

18. Atlas topographique de la Suisse 1:50.000 (carte Siegfried), Orsières, feuille ${ }^{\circ} 529,1878$.

19. Andenmatten et Paccolat (2010:311-313) ; Andenmatten et Pignolet (2015a : 525-527) ; Andenmatten et Pignolet (2015b, 297-299); Andenmatten et Pignolet (2016b : 226-228).

20. Pour une synthèse de l'étude de l'inscription : Aberson et al. (2021:309-332). 
Autour du Mur (dit) d'Hannibal : appréhender un « dispositif militaire » du premier siècle av. J.-C. dans les

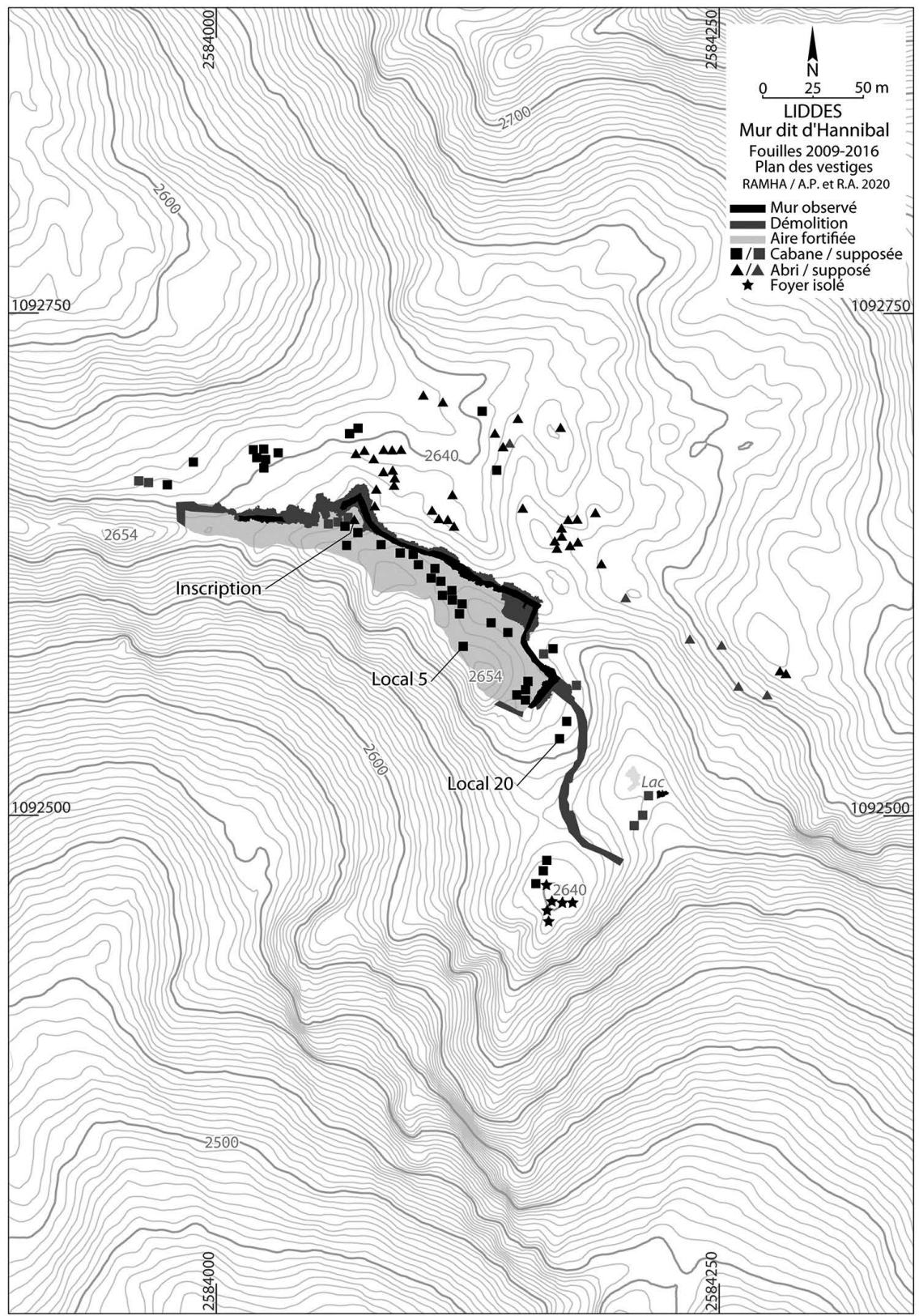

Figure 2. Mur (dit) d'Hannibal, Plan des vestiges (C) RAMHA 2020).*

* Les coordonnées géographiques notifiées sur les plans utilisent le système suisse CH1903+. 
extérieures permettent de définir que la date la plus probable d'occupation se situe entre 45 et 16/15 avant J.-C. ${ }^{21}$

Les 21 datations par le radiocarbone réalisées sur le site et mises en regard du mobilier permettent également de très intéressantes observations d'ordre méthodologique. En effet, toutes les dates obtenues sur des macrorestes végétaux carbonisés (par exemple : vicia faba ou hordeum) et celles sur des charbons de bois jugés favorables par l'anthracologue du projet ${ }^{22}$ (par exemple : alnus viridis, maloideae, prunoideae ou fragments de branches) se situent dans une fourchette entre le deuxième siècle avant J.-C. et le milieu du premier siècle après J.-C. À défaut, des datations ont aussi été réalisées sur des charbons de bois jugés moins favorables ${ }^{23}$ végétaux à longue durée de vie (par exemple : pinus, picea ou larix) et ces dernières donnent des dates en moyenne plus anciennes, entre le quatrième et le milieu du premier siècle avant J.-C. L'analyse stratigraphique permet cependant l'association de mobilier daté entre
70/60 et 16/15 avant J.-C. avec des datations radiocarbones parmi les plus jeunes mais aussi les plus anciennes. Enfin, dans le local 5, un charbon de bois daté entre le deuxième et le premier siècle avant J.-C. ${ }^{24}$ est stratigraphiquement situé au-dessus d'une fève et de mobilier à dater au plus tôt du second tiers du premier siècle avant J.-C. Un effet (dit) « vieux-bois » des datations sur charbon " moins favorables" doit donc être pris en considération. ${ }^{25}$

Des quatre monnaies romaines mises au jour, seul un as républicain très usé, frappé à Rome entre 211 et 146 avant J.-C., a été retrouvé dans un contexte associé à un niveau d'occupation. ${ }^{26}$ Les trois autres monnaies postérieures proviennent de zones de cheminement. ${ }^{27}$

Le corpus mobilier mis au jour peut être associé à une tradition indigène pour la céramique, une partie de la parure et de rares éléments associés à l'armement tandis que la catégorie la plus représentée est celle des objets qui sont communément associés aux militaria romains, dont une cen-

21. Le local 005 est situé à l'intérieur de la zone protégée par l'enceinte sur le site du Mur (dit) d'Hannibal. L'intérieur de ce bâtiment présentait en alternance plusieurs niveaux de circulation et de défournement de son foyer central. Le terminus post quem de l'occupation du local est de 45 avant J.-C. sur la base d'une datation par le radiocarbone de son niveau de défournement le plus ancien : BE-11640.1.1, foyer US103, macroreste (vicia faba), 45calBC (95.4) 53calAD; OxCal v4.3.2 (Bronk Ramsey, 2017), r5, IntCal13 atmospheric curve (Reimer et al., 2013). Son terminus ante quem n'est pas postérieur à 16/15 avant J.-C. sur la base du diamètre des clous de chaussures retrouvés dans le bâtiment (Fig. 3 : HA15081, HA15-096, HA15-097 et HA15-115). Quant à la céramique mise au jour conjointement aux clous de chaussures romains dans le local, elle est uniquement de tradition indigène (HA15-099 et HA15-101 identification sur la base des pâtes, non représentés).

22. Alexa Dufraisse, Muséum national d'Histoire naturelle.

23. Certains charbons analysés dans le cadre des études préliminaires permettent de restituer la récolte de bois d'un diamètre minimum jusqu'à $20 \mathrm{~cm}$. Ces dimensions laissent envisager l'abattage d'arbres âgés (Aberson et Andenmatten, 2021, à paraitre).

24. BE-11639.1.1, foyer US103, charbon de bois (maloideae), 175 (95.4\%) 47calBC; OxCal v4.3.2 (Bronk Ramsey, 2017), r5, IntCal13 atmospheric curve (Reimer et al., 2013).

25. Pour un développement méthodologique de la question se référer à la bibliographie relativement volumineuse sur le sujet depuis Schiffer (1986).

26. HA14-034.

27. HA16-220, Auguste, Lyon, as, 9-14 après J.-C., HA16-221, Claude, Rome, as, 41-54 après J.-C. et HA15-139, Gordien III, Rome ou Antioche, antoninien, 238-240. 


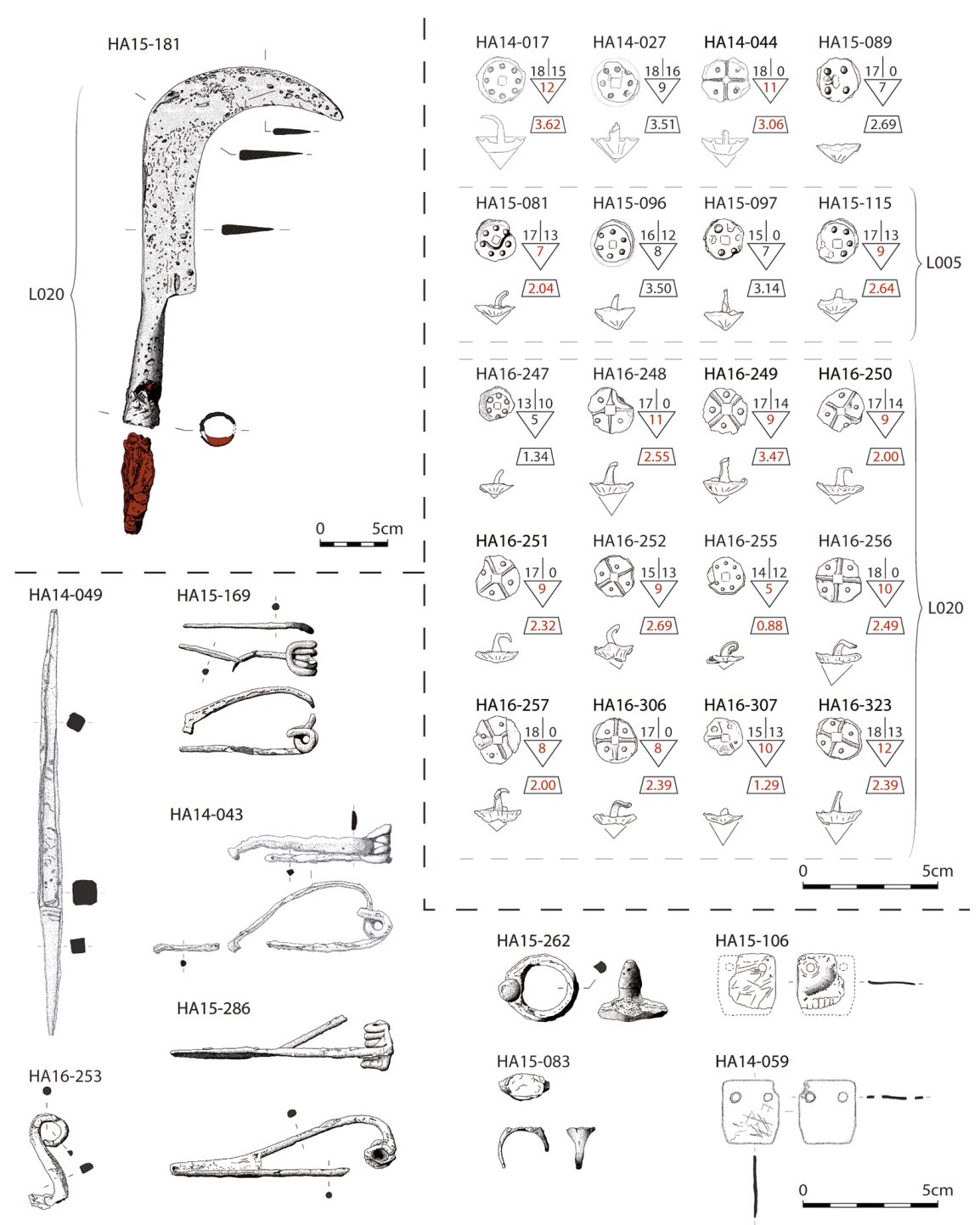

Figure 3. Mur (dit) d'Hannibal, Planche présentant une sélection du mobilier métallique récolté sur le site, P.-E. Mottiez et R. Andenmatten (C) RAMHA 2020) : HA15-181, fer et bois, serpe ; HA14-017, HA14-027, HA14-044, HA15-089, HA15-081, HA15-096, HA15-097, HA15-115, HA16-247, HA16-248, HA16-249, HA16-250, HA16-251, HA16-252, HA16255, HA16-256, HA16-257, HA16-306, HA16-307, HA16-323, fer, clous de chaussures ; HA14-049, fer, pointe de trait de type (dit) «Numance»; HA14-043, HA15-169, HA15-286, fer, fibules de schéma La Tène finale ; HA15-262, alliage cuivreux, anneau de ceinturon ; HA15-083, fer, bague à intaille ; HA16-253, fer, fragment de porte-cimier ? ; HA14-059, HA15-106, alliage cuivreux, écaille d'armure. 


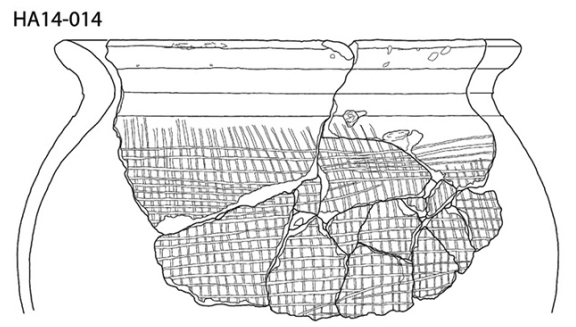

HA14-022
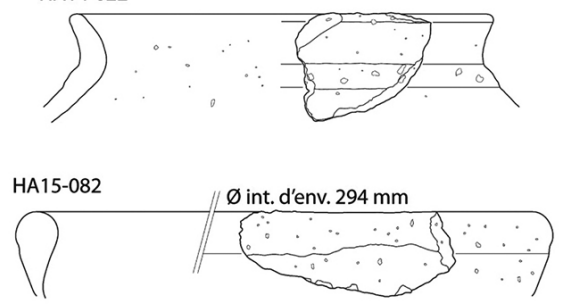
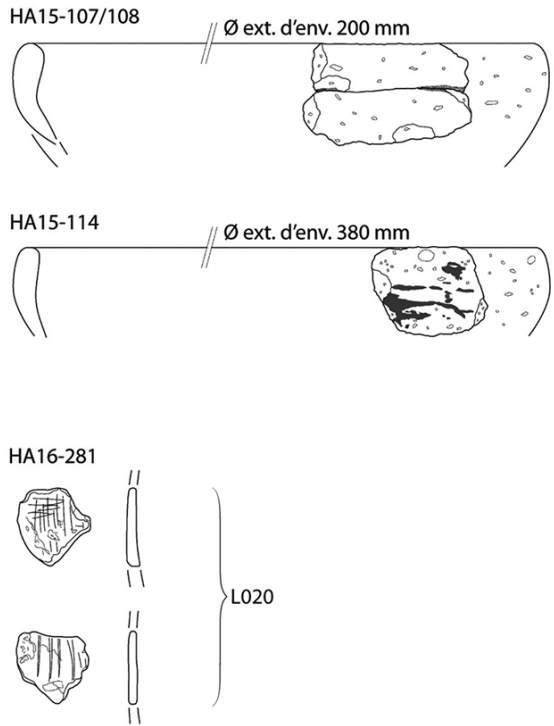

0 $5 \mathrm{~cm}$

Figure 4. Mur (dit) d'Hannibal, Planche présentant une sélection du mobilier céramique récolté sur le site, P.-E. Mottiez et R. Andenmatten (C RAMHA 2020) : HA14-014, céramique grossière tournée, pot à cuire avec bord déversé, lèvre amincie et décor au peigne ; HA14-022, céramique grossière modelée (dite) indigène, pot à cuire avec bord déversé et lèvre amincie ; HA15-082, céramique grossière tournée, jatte à bord épaissi légèrement rentrant et lèvre arrondie ; HA15-107/108, céramique grossière modelée (dite) indigène, jatte à bord faiblement épaissi, redressé et légèrement rentrant avec une lèvre arrondie ; HA15-114, céramique grossière (dite) indigène (probablement modelée), jatte à bord faiblement épaissi, redressé et légèrement rentrant avec une lèvre arrondie ; HA16-281, céramique grossière modelée (dite) indigène, fragments de panse (probablement pot à cuire).

taine de clous de chaussures et une serpe. ${ }^{28}$ Des ensembles de matériel mixte ont ainsi été repérés dans un même niveau à l'intérieur de bâtiments et des interférences graphémiques et linguistiques latines ont été observées dans l'inscription «indi- gène». ${ }^{29}$ À ce stade et en l'absence de sériation entre plusieurs occupations, indigènes puis romaines ou romaines puis indigènes, l'hypothèse favorisée est celle d'une position occupée par des auxiliaires indigènes de l'armée romaine.

28. La serpe HA15-181 doit être considérée comme un outil qui pourrait servir d'arme occasionnelle, d'une manière similaire aux outils pionniers des militaires du $20^{\mathrm{e}}$ siècle. Pour la période républicaine et les débuts du Haut-Empire, il semble que la plupart de ces serpes de grandes dimensions proviennent de lieux de cantonnement militaire ou de combats (Pohanka, 1986 : 179; Martin-Kilcher, 2011 : 36 ; Ribera i Lacomba et Calvo Galvez, 1995 : 30). 29. Aberson et al. (2021:309-332).

29. Aberson et al. (2021:309-332). 
Autour du Mur (dit) d'Hannibal : appréhender un "dispositif militaire " du premier siècle av. J.-C. dans les

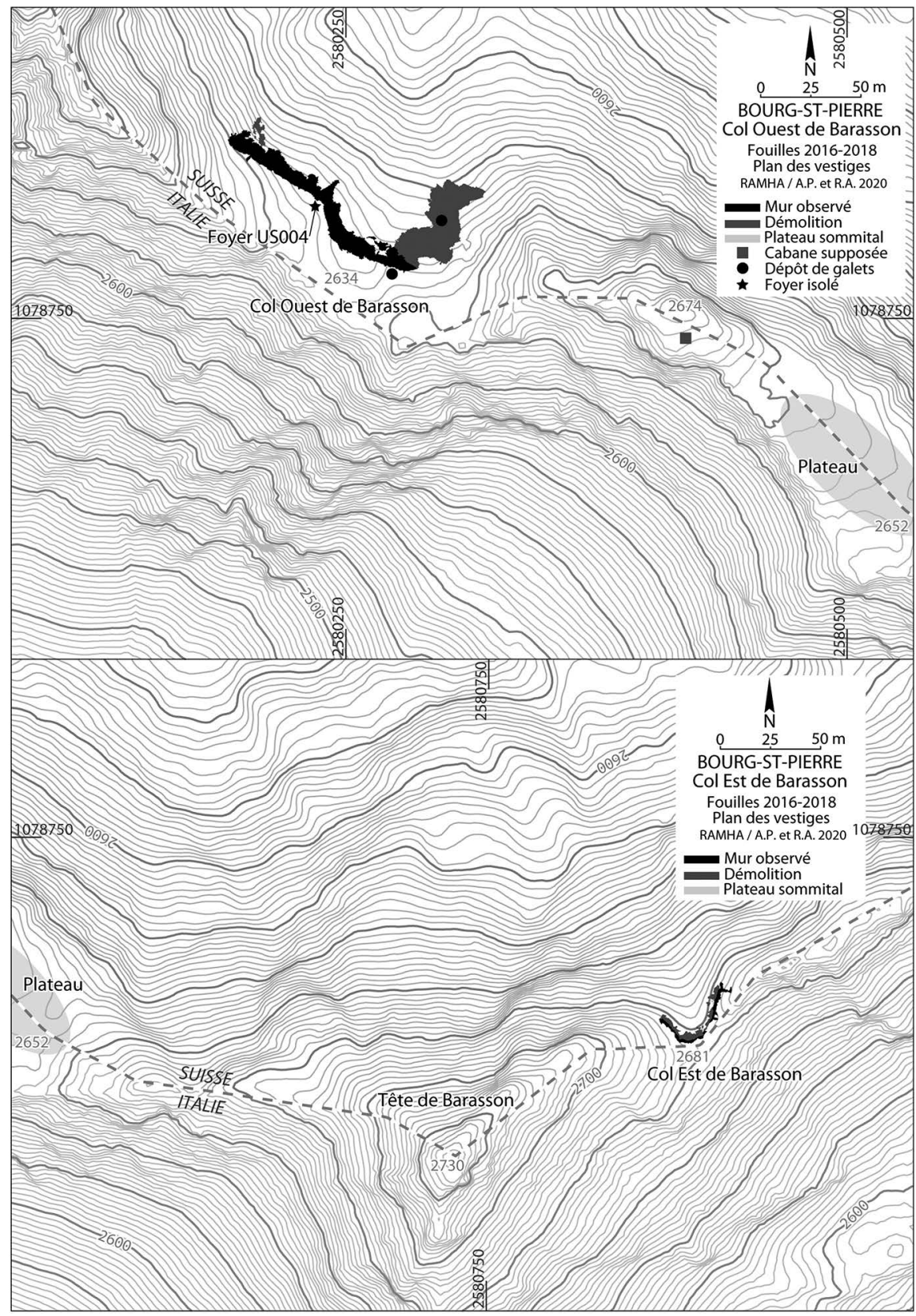

Figure 5. Cols de Barasson, Plan des vestiges, (C) RAMHA 2020. 


\subsection{Les Cols de Barasson (Bourg-Saint-} Pierre, Valais, Suisse, Saint-Rémy-enBosses et Saint-Oyen, Vallée d'Aoste, Italie, 2634 et $2681 \mathrm{~m}$ )

Les positions fortifiées des Cols de Barasson se situent sur deux passages à l'ouest et à l'est de la Tête de Barasson, une éminence de l'arête rocheuse de la frontière italo-suisse. À cheval sur la limite entre les deux pays, un grand plateau est également présent sur l'arête entre le Col Ouest et la Tête de Barasson. Il n'est aisément accessible que par l'arête et offre une surface de près de $7000 \mathrm{~m}^{2}$.

Ces emplacements avaient déjà été relevés sur des cartes du $18^{\mathrm{e}}$ siècle, ${ }^{30}$ mais aucune interprétation n'en était proposée. La voie de passage conduisant au Col Ouest ainsi que le col lui-même avaient fait l'objet de prospections en 2006 et un clou de chaussure romain y avait été mis en évidence. La fortification avait intrigué les chercheurs mais sans qu'ils aient la disponibilité pour effectuer de plus amples recherches. ${ }^{31}$

Une semaine et demie d'investigations a été conduite sur ces positions avec cinq personnes en 2016 et une journée de prospections communes avec une équipe de dix personnes a été réalisée en complément sur l'arête frontière en 2018. Des relevés de surface, des prospections ciblées au détecteur de métaux et trois petits sondages ont ainsi été réalisés. ${ }^{32}$

Le Col Ouest de Barasson est situé à $2634 \mathrm{~m}$ d'altitude en un point où l'arête frontière présente une pente moyenne $(<$ de $35 \%)$ sur son versant nord et des barres rocheuses sur son versant sud. Ce dernier est ainsi naturellement défendu. Un mur de près de $200 \mathrm{~m}$ de long et jusqu'à $2 \mathrm{~m}$ de haut ferme le dispositif défensif du côté nord à une trentaine de mètres à l'aval de l'arête rocheuse et délimite ainsi un premier espace protégé d'env. $3500 \mathrm{~m}^{2}$. Cette zone soumise quasi quotidiennement à des vents violents semble cependant moins favorable à des installations que le grand plateau situé plus à l'est sur l'arête. On ne peut relever sur ce premier col que la présence d'un foyer et de deux dépôts de galets, ${ }^{33}$ à la base du parement intérieur de la fortification.

Une datation par le radiocarbone sur un charbon de bois, ${ }^{34}$ ainsi qu'un clou de chaussure $^{35}$ permettent de dater le foyer susmentionné entre le deuxième quart du premier siècle avant J.-C. et 16/15 avant J.-C. Il est installé contre le parement amont du mur et la démolition de ce dernier le recouvre. La structure de combustion est donc postérieure à la construction

30. Plan géométrique du Grand-Saint-Bernard par Durieu et de Rivaz en 1762 (Archives de l'Etat du Valais, Confinia 2/3/5).

31. Benedetti et Curdy (2008: 385-386).

32. Andenmatten et Pignolet (2016b : 228-230).

33. Il s'agit de galets de rivière sélectionnés dont les provenances sont en cours d'évaluation. Ces éléments sont naturellement absents de la position et y ont nécessairement été amenés par l'homme. Leurs formes arrondies indiquent des probables origines alluviales en fond de vallée.

34. BE-11647.1.1, foyer US004, charbon de bois (picea / larix), 198 (87.1\%) 87calBC / 79 (8.3\%) 56calBC; OxCal v4.3.2 (Bronk Ramsey, 2017), r5, IntCal13 atmospheric curve (Reimer et al., 2013).

35. Ce clou de chaussure (Fig. 6 : BBO16-007) possède un diamètre de $16 \mathrm{~mm}$ et a été retrouvé dans le niveau d'utilisation du foyer US004. Il permet de proposer pour cette structure un terminus post quem de 70/60 avant J.-C. et un terminus ante quem qui n'est pas postérieur à 16/15 avant J.-C. (Fig. 13 et Martin-Kilcher, 2021, à paraître). La datation par le radiocarbone BE-11647.1.1 illustrerait alors un « effet vieux bois » tout à fait plausible au vu de l'essence analysée. 
et antérieure à l'abandon de la structure défensive. Une partie du reste du mobilier récolté sur le site s'intègre également dans une fourchette chronologique similaire. ${ }^{36}$ Cependant, un objet isolé peut être rattaché à l'âge du Bronze moyen et plusieurs éléments illustrent la poursuite de l'utilisation ou de l'occupation de ce passage entre l'Antiquité et l'époque contemporaine. Une cabane, dans laquelle sont conservés des restes de charpente en bois, correspond très probablement aux vestiges de la mobilisation de 1939-1945 et un pylône de ligne à haute tension est installé sur le bord oriental du col vers 1950. Les mises à terre de ce dernier ainsi que les aménagements pour sa construction ont parfois perturbé le soussol du secteur en profondeur.

Sur le grand plateau situé entre le Col Ouest et la Tête de Barasson à une altitude moyenne de $2655 \mathrm{~m}$ et sur l'arête qui y conduit depuis le col occidental, des replats offrent des opportunités pour des installations. Un seul potentiel fond de bâtiment a cependant pu être repéré sur l'arête tandis que d'éventuels aménagements sur le plateau sont difficiles à appréhender au vu des nombreux vestiges associés à la construction de deux pylônes pour une seconde ligne à haute tension vers 1965 . Une datation par le radiocarbone sur un charbon de bois ${ }^{37}$ permet de rattacher le premier état du probable fond de bâtiment situé sur l'arête au grand mur de barrage et au foyer mis au jour sur le col occidental.
Les objets documentés dans ce secteur sont, par contre, très majoritairement d'époque contemporaine et du 20e siècle ou alors indéterminés. Une seconde datation par le radiocarbone d'un niveau d'occupation sur l'arête à quelques mètres du plateau ainsi que la présence de restes de bois très érodés en surface peuvent probablement être associés à la seconde guerre mondiale ou à la construction des pylônes électriques. ${ }^{38}$

Le col oriental se situe à env. $350 \mathrm{~m}$ à l'est du plateau, entre la Tête et la Pointe de Barasson. Son altitude est de $2681 \mathrm{~m}$ et son accès nord passe par une combe étroite et raide dont les derniers mètres présentent un dénivelé moindre. Plusieurs petites barres rocheuses limitent les possibilités de passage sur le versant sud du col qui possède une pente générale plus raide.

Un mur d'une cinquantaine de mètres de longueur avec une hauteur conservée d'env. $1.5 \mathrm{~m}$ vient barrer l'accès au col dans la combe nord en suivant une courbe de niveau. Aucun aménagement ne peut directement être associé à la période d'édification de cette structure. Plusieurs murets construits moins soigneusement, en partie sur le mur de fortification, et délimitant des abris ou soutenant un chemin, peuvent probablement être associés à la mobilisation du second conflit mondial et non à l'Antiquité. Directement à l'amont du mur principal, deux clous de chaussures tardo-républi-

36. C'est le cas des clous de chaussures BBO16-013, BBO16-014 et BBO16-068 (Fig. 6) ainsi que de la pointe (dite) de "type Numance » $\mathrm{BBO} 16-067$ (Fig. 6). Il est par contre très probable que le clou BBO16-027 (Fig. 6) corresponde à un passage postérieur.

37. BE-11648.1.1, niveau d'occupation US008, charbon de bois (essence non-déterminée), 186 (95.4\%) 52calBC; OxCal v4.3.2 (Bronk Ramsey, 2017), r5, IntCal13 atmospheric curve (Reimer et al., 2013).

38. BE-11649.1.1, niveau d'occupation US010, charbon de bois (essence non-déterminée), $1680(33.2 \%)$ 1764calAD / 1801 (47.4\%) 1893calAD / 1907 (14.8\%) 1939calAD / Out of range ; OxCal v4.3.2 (Bronk Ramsey, 2017), r5, IntCal13 atmospheric curve (Reimer et al., 2013). 
cains ainsi qu'un fragment de ressort de fibule ont été récoltés. ${ }^{39}$ Ces derniers se trouvaient en partie associés au colluvionnement d'un niveau d'occupation charbonneux et permettent de proposer une datation de l'occupation principale du col oriental similaire à l'arête et au col occidental.

Les positions fortifiées du fond de la combe de Barasson présentent donc des similitudes mais aussi des différences avec le site du Mur (dit) d'Hannibal. Les datations par le radiocarbone ainsi que le mobilier permettent d'identifier des occupations datées de la même période à ces endroits. Structurellement, les murs défensifs sont également très proches. Le mur du Col Ouest de Barasson permet, en utilisant les défenses naturelles offertes par le terrain, d'assurer une sécurité à $360^{\circ}$ pour une zone de près de $10^{\prime} 500 \mathrm{~m}^{2}$ (amont du mur, arête et plateau). L'espace protégé semble cependant très peu occupé et ne pas avoir fait l'objet d'installations aussi développées qu'au Mur (dit) d'Hannibal. Il pourrait ainsi s'agir d'une position préparée mais occupée seulement par une troupe réduite, voire pendant peu de temps, ou d'un site dont les occupants n'ont pas mis en ouvre des cabanes et ont utilisé des structures plus légères, éventuellement des tentes. La présence de plusieurs stocks de pierres de jet ${ }^{40}$ le long du parement intérieur du mur principal est une particularité du Col Ouest de Barasson qui n'a été repérée sur aucun des autres sites valaisans. ${ }^{41}$ Les galets de Barasson possèdent des dimensions moyennes supérieures et semblent moins bien calibrés que ceux mis en évidence sur d'autres positions de l'âge du Fer ou tardo-républicaines. ${ }^{42}$ Ils correspondent cependant clairement à des projectiles et la mention de l'utilisation de pierres de jet par les indigènes valaisans chez César durant la bataille d'Octodure doit être relevée. ${ }^{43}$ La découverte d'un galet similaire dans un petit lac situé $150 \mathrm{~m}$ à l'aval, au nord, du mur de Barasson ouest interpelle. Un essai de tir dans le plan d'eau a été évoqué mais cette explication demeure purement hypothétique.

Le Col Est de Barasson n'offre qu'un espace très limité pour s'installer (moins de $350 \mathrm{~m}^{2}$ ) et peut certainement être considéré comme une position de barrage annexe à la position principale. Il est en effet aussi possible de le rejoindre par l'arête depuis le grand plateau de Barasson ouest.

On ne peut, de par la faible ampleur des investigations et le peu de mobilier ainsi que de structures découverts, définir avec exactitude qui étaient les occupants de ces positions. Â ce jour, il faut y relever l'absence de marqueurs indigènes, si ce n'est le fragment de fibule et une présence de clous de chaussures associés à des mili-

39. Clous de chaussures BBE16-030 et BBE16-031 ainsi que ressort de fibule BBE16-026 (Fig. 6).

40. Dimensions moyennes de $75.45 \mathrm{~mm}$ par $62.4 \mathrm{~mm}$ pour les 150 galets repérés : 113 galets d'une longueur de moins de $90 \mathrm{~mm}, 20$ galets présentant des longueurs entre 90 et $100 \mathrm{~mm}$ ainsi que 17 galets mesurant entre 100 et $125 \mathrm{~mm}$ de long. Deux stocks principaux ainsi que quelques galets isolés ont été documentés. Certains se trouvaient à proximité du foyer investigué.

41. Les dépôts de galets observés sur les sites de la Punta Fetita et de la Tour Ronde en Vallée d'Aoste semblent présenter des dimensions moindres et une calibration plus régulière.

42. Selon Deyber et al. (2018 : 21-27), les moyennes des longueurs des galets par amas du Lampourdier oscillent entre 41.5 et $57.7 \mathrm{~mm}$. Seuls 38 des 150 galets du Col Ouest de Barasson entrent dans ces dimensions.

43. Des lapides gaesaque sont mentionnés lors de la Bataille d'Octodure (César, BG 3, 4, 1). 

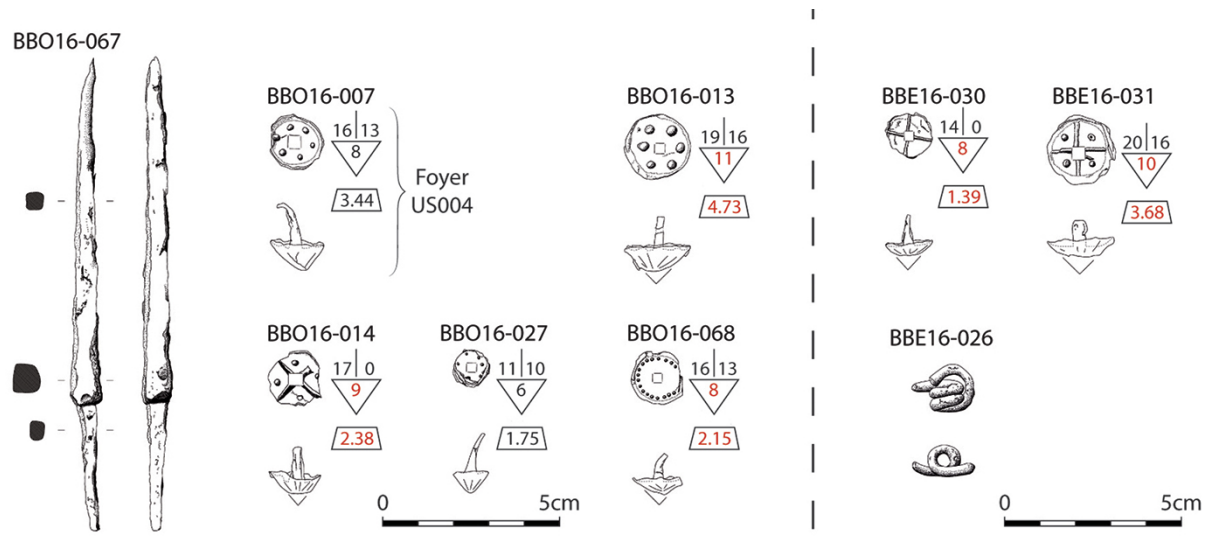

Figure 6. Cols de Barasson, Planche présentant une sélection du mobilier métallique récolté sur les sites, P.-E. Mottiez et R. Andenmatten, (C) RAMHA 2020, Col Ouest de Barasson : BBO16067, fer, pointe de trait de type (dit) «Numance "; BBO16-007, BBO16-013, BBO16-014, BBO16-027, BBO16-068, fer, clous de chaussures et Col Est de Barasson : BBE16-030, BBE16031, fer, clous de chaussures ; BBE16-026, fer, fragment de fibule de schéma La Tène finale.

taria tardo-républicains. On supposerait ainsi volontiers qu'il s'agisse d'une position occupée par une troupe romaine dont la nature, auxiliaire ou légionnaire, reste à définir.

\subsection{Le Col d'Annibal (Bourg-Saint- Pierre, Valais, Suisse, Etrouble, Vallée d'Aoste, Italie, 2995 à 3059 m)}

Le Col d'Annibal est le passage le plus oriental entre le Val d'Entremont (Valais, Suisse) et la Vallée du Grand-Saint-Bernard (Vallée d'Aoste, Italie). Des aménagements y ont été repérés en discontinu le long de l'arête frontière sur près de $350 \mathrm{~m}$ en partant du point bas au niveau du col à $2995 \mathrm{~m}$ pour s'arrêter sous le sommet de la Testa Grisa à $3059 \mathrm{~m}$. Le versant sud de l'arête rocheuse est formé d'éboulis instables avec une pente très raide. Le versant nord de l'arête surplombe le Glacier de Proz, ses moraines, les éboulis de ces dernières et des névés situés dans les creux des zones de tassement à la périphérie du glacier en retrait. L'accès au col, même s'il nécessite de passer dans des zones englacées, est plus aisé par le nord que par le sud.

La première mention de l'observation de ruines ainsi que de la découverte d'une pointe de lance à cet emplacement datent de $1901 .{ }^{44}$ En 2007, dans le cadre du programme de prospections conduit également au Col Ouest de Barasson, le mur barrant le col a fait l'objet d'un relevé photographique et quelques aménagements frustes ont été repérés sur le flanc sud de l'arête. ${ }^{45}$ Aucun élément archéologique n'a été découvert à cette occasion. En 2013, des premiers bois travaillés sont repérés et récupérés par une équipe de

44. Cibrario (1901:386).

45. Benedetti et Curdy (2008: 387). 


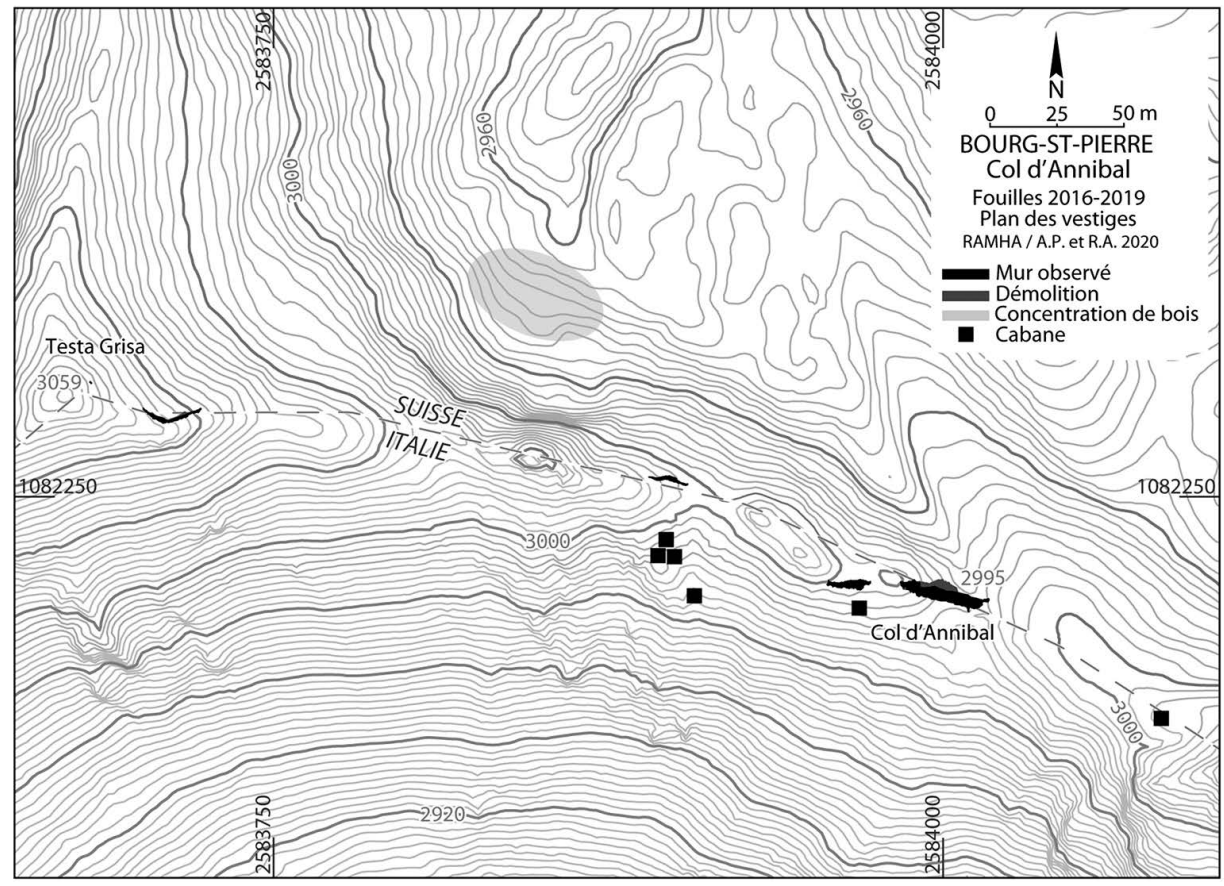

Figure 7. Col d'Annibal, Plan des vestiges (C) RAMHA 2020).

glaciologues de l'université de Fribourg sous la direction de Reynald Delaloye. Rapidement ces artefacts intéressent l'archéologue Philippe Curdy et le glaciologue Ralph Lugon qui mènent un projet de recherche en archéologie glaciaire et une journée de prospection est ainsi organisée en 2014.

En 2016, notre groupe de recherche reprend les prospections visuelles et au détecteur de métaux sur le versant valaisan ainsi que la documentation des vestiges lors d'une campagne d'une semaine avec cinq personnes. En 2019, enfin, des prospections communes sont organisées sur l'arête frontière, plusieurs bâtiments repérés lors de la campagne précédente sont documentés et 58 nouveaux fragments travaillés de bois sont récupérés.
La structure la plus imposante du site est le mur du col avec une longueur d'env. $25 \mathrm{~m}$ et une hauteur d'env. $2.1 \mathrm{~m}$ du côté de son parement nord. En suivant l'arête depuis le col en direction de l'ouest, quatre autres tronçons de murs qui mesurent respectivement $11 \mathrm{~m}, 13 \mathrm{~m}, 25 \mathrm{~m}$ et $1 \mathrm{~m}$ de longueur pour une hauteur conservée jusqu'à $1 \mathrm{~m}$ ont été aussi repérés. Ces derniers renforcent des points de passage aisés de l'arête et leur état de conservation moins bon que le mur du col peut s'expliquer par leurs situations plus exposées en sommet du relief ou dans des pentes raides. Ces structures permettent d'envisager un dispositif linéaire de près de $350 \mathrm{~m}$ barrant l'arête.

À côté des nombreux éventuels refuges contre des blocs sans aménagement 
particulier, 6 petites constructions doivent être relevées. Il s'agit de 5 fonds de cabanes situés sur le versant sud de l'arête et d'une cabane double construite sur la moraine recouvrant la crête au sud-est du site.

En contrebas des murs sur le versant nord se trouve le principal secteur de découverte de fragments de bois travaillés. Il s'agit de 2 fragments d'une poutrelle équarrie, de 1 fragment de planchette, de 1 fragment de tronc fendu, de 4 fragments de formes indéterminées et de 152 fragments de perches, de $0.1 \mathrm{~m}$ à $1.85 \mathrm{~m}$ de longueur pour un diamètre moyen de $2 \mathrm{~cm}$. 48 de ces résineux ébranchés possèdent l'une ou les deux extrémités appointies. Deux interprétations en ont été proposées. Il pourrait s'agir de perches pour marquer un cheminement dans un secteur englacé, ${ }^{46}$ mais également d'éléments ayant participé au renforcement de la crête sous la forme d'une barricade de piquets appointis positionnés sur ou au-devant des murs, voire pour en compléter un tronçon sur une portion d'arête recouverte de glace.

À l'exception des bois et du fer de lance découvert en 1901, et perdu aujourd'hui, aucun mobilier antique n'a pu être mis au jour sur le site lors de nos investigations. Le sol morainique très vacuolaire est totalement lessivé et ne permet pas la conservation in situ de petits objets ou de niveaux d'occupation. Ces derniers s'infiltrent probablement rapidement dans les amas de blocs qui constituent les moraines anciennes et les éboulis. ${ }^{47}$

Il nous est dans ces conditions difficile de proposer une datation des structures repérées. Les 5 fonds de cabanes observés à l'arrière des murs ainsi que ces derniers présentent cependant des états de conservation relativement similaires qui laissent envisager une contemporanéité. La cabane double située au sud-est semble quant à elle être plus récente. Des réoccupations peuvent cependant aussi être envisagées dans cette zone.

Tous les bois retrouvés sur le versant nord de l'arête présentent des caractéristiques proches. 7 datations par le radiocarbone sur ces derniers entrent dans une fourchette de 166 av. J.-C. à 121 ap. J.-C. Le bois présentant le terminus ante quem le plus ancien est l'un des fragments de poutrelle équarrie daté avant 49 av. J.-C. ${ }^{48}$ tandis que le fragment donnant le terminus post quem le plus récent, en 40 av. J.-C. ${ }^{49}$, est l'une des perches à extrémité appointie. Une utilisation de la position ou tout au moins du passage durant la période d'occupation du site du Mur (dit) d'Hannibal peut donc être envisagée pour ce site.

Malgré leurs petits diamètres, les fragments de perches documentés semblent posséder assez de cernes et sont assez nombreux pour envisager une dendrochronologie relative ainsi qu'un éventuel

46. Il s'agit de l'hypothèse développée par Philippe Curdy sur la base du texte d'Ammien Marcellin, Res gestae, $15,10,5$.

47. Dans ce contexte périglaciaire, moraines et éboulis sont aussi en mouvement permanent. Des fragments de bois ont d'ailleurs été documentés sous des amas de blocs qui les ont recouverts au fil du temps.

48. ETH-74887, fragment de poutrelle équarrie, BCA16-008, bois (abies), 166 (95.4\%) 49calBC; OxCal v4.3.2 (Bronk Ramsey, 2017), r5, IntCal13 atmospheric curve (Reimer et al., 2013).

49. Poz-59850, fragment de perche à extrémité appointie, HANN13-001, bois (essence non-déterminée), 40calBC (91.9\%) 87calAD / 105 (3.5\%) 121 calAD ; OxCal v4.3.2 (Bronk Ramsey, 2017), r5, IntCal13 atmospheric curve (Reimer et al., 2013). 


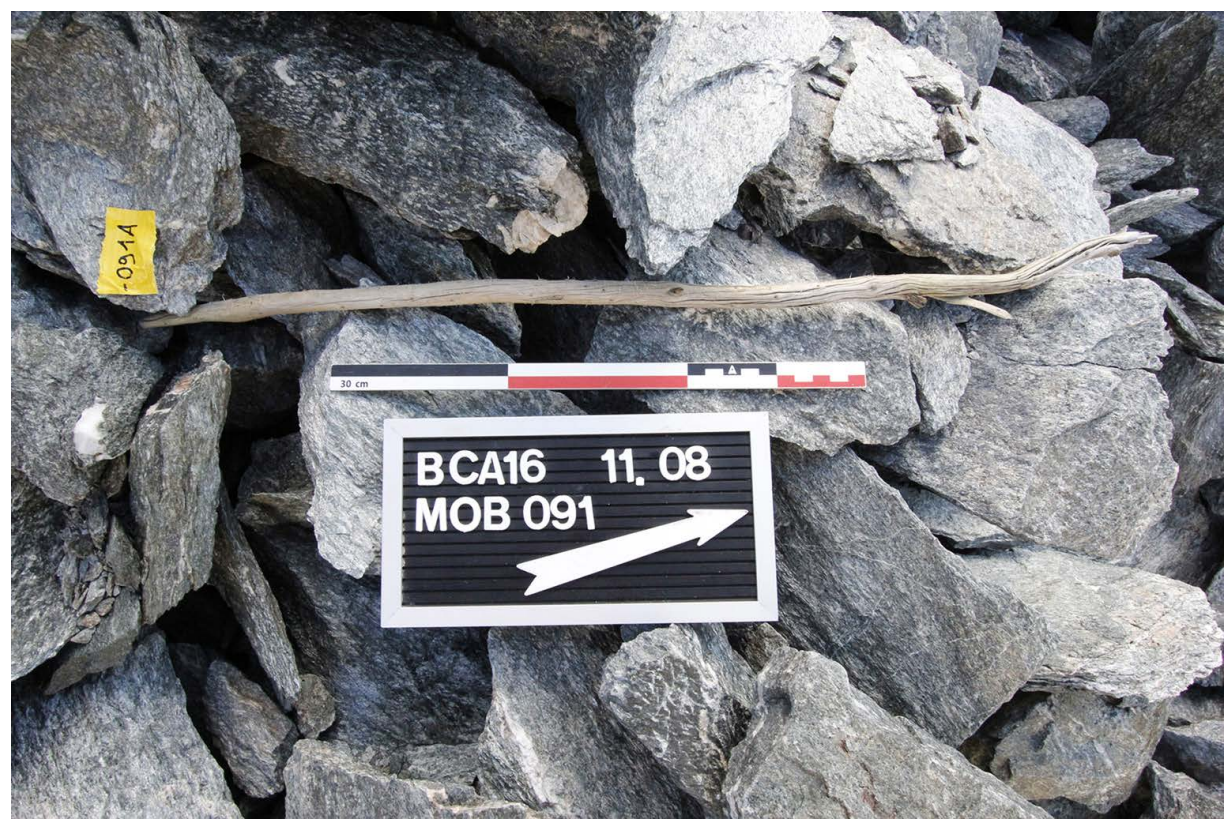

Figure 8. Col d'Annibal, Fragment de perche appointie (C) RAMHA 2016).

rattachement sur une courbe absolue avec le soutien des datations par le radiocarbone. L'étude a été débutée en 2019 au Laboratoire de dendrochronologie de Neuchâtel ${ }^{50}$ et des résultats devraient pouvoir être présentés prochainement.

\subsection{Le Plan de Tcholeire (Bourg-Saint- Pierre, Valais, Suisse, 2465 m)}

Les nombreux fonds de cabanes et quelques abris du Plan de Tcholeire sont répartis en cinq secteurs éloignés les uns des autres d'au moins une centaine de mètres. Ils ont été repérés la première fois en 2016 par des membres du groupe scientifique RAMHA en excursion et ont fait l'objet d'investigations préliminaires lors de deux journées avec une équipe de huit personnes en $2018 .{ }^{51} \mathrm{Il}$ s'agissait principalement d'effectuer le levé d'un plan général de tous les aménagements repérés, de prospecter quelques secteurs clés et de réaliser des sondages de petites dimensions dans des structures sélectionnées.

Les deux noyaux d'occupation principaux comptent chacun une vingtaine de structures avec une majorité d'espaces avec un solin périphérique d'un à deux niveaux de pierres et quelques abris contre ou sous des blocs. Entre ces deux secteurs et légèrement en direction de l'est, un noyau plus petit, comprenant 5 cabanes et 3 abris, est isolé dans une moraine. Plus au sud-ouest, deux grappes de 5 cabanes ont également été repérées. Le

50. Fabien Langenegger, Laboratoire de dendrochronologie de Neuchâtel.

51. Andenmatten et al. (2020, à paraittre). 
Autour du Mur (dit) d'Hannibal : appréhender un " dispositif militaire » du premier siècle av. J.-C. dans les

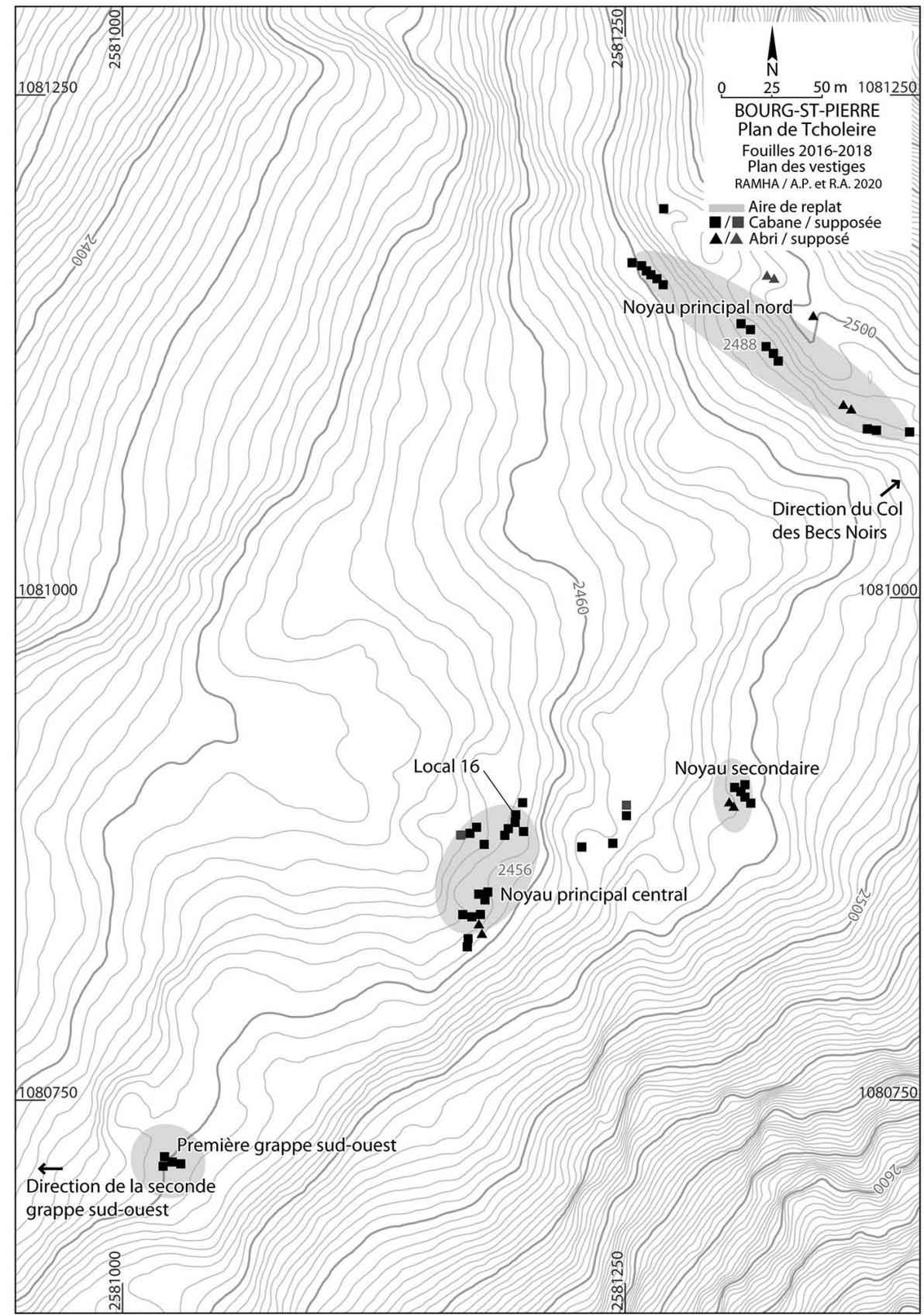

Figure 9. Plan de Tcholeire, Plan des vestiges, (C) RAMHA 2020. 
noyau d'occupation principal nord est installé sur et au pied des éboulis d'un cordon morainique lessivé entre 2480 et $2500 \mathrm{~m}$ d'altitude. Les aménagements sont organisés principalement sur deux niveaux avec un alignement inférieur de " cabanes " qui occupe le pied des éboulis et quelques abris et " cabanes " supérieurs qui sont aménagés à l'arrière de la ligne de crête du cordon morainique. Le tout se développe sur $200 \mathrm{~m}$ en longueur pour une trentaine de mètres en largeur. À l'exception d'un alignement de 5 "cabanes " contiguës sur le niveau inférieur au nordouest, l'organisation est plutôt lâche.

Le second noyau principal, au centre de la zone, se situe à la limite entre des affleurements de la roche-mère et des cônes ou des langues d'éboulis à env. $2450 \mathrm{~m}$ d'altitude. Les aménagements s'y développent principalement en deux zones de densité moyenne sur un secteur total de 75 par 50 m et sont accompagnés de quelques fonds de cabanes plus isolés. Le troisième noyau d'occupation, plus petit, est situé dans des éboulis à env. $2480 \mathrm{~m}$ d'altitude. Il est dense et occupe une surface de moins de $100 \mathrm{~m}^{2}$. Les grappes repérées au sud-ouest sont, enfin, aussi installées dans des éboulis ou à leurs limites avales. Elles se situent respectivement à une altitude d'env. $2460 \mathrm{~m}$ pour la moins éloignée et à env. $2430 \mathrm{~m}$ pour la plus à l'ouest.

Par leurs dimensions et leurs qualités d'exécution, il a été immédiatement observé que les aménagements du Plan de Tcholeire étaient plus proches des abris et des fonds de cabanes frustes des moraines extérieures du site du Mur (dit) d'Hannibal que des bâtiments associés à son enceinte. Si une partie des structures situées dans des moraines semblaient totalement lessivées, d'autres, dans des secteurs recouverts d'humus, laissaient envisager la conservation de niveaux d'occupation ou de circulation. À une exception près, les structures n'en ont cependant pas livré et seul le local 16 dans le noyau central a livré un niveau charbonneux. De manière similaire, les abris et les fonds des cabanes des moraines du Mur (dit) d'Hannibal n'ont pas livré de niveaux conservés et, à une exception près, aucun mobilier datant n'y a été découvert.

Quelques objets ont aussi été retrouvés à proximité ou dans le périmètre de certaines structures mais toujours dans l'humus et jamais en association avec un niveau d'occupation ou de circulation. C'est entre autres le cas de plusieurs billes en plomb retrouvées sur l'ensemble du site et qui correspondent probablement à des restes de sous-munition. ${ }^{52}$ Parmi les éléments mis au jour, on relèvera aussi une monnaie romaine du troisième siècle ap. J.-C. dans l'humus formé sur une dalle à l'intérieur d'une cabane de la seconde grappe sud-ouest, un fer à cheval probablement daté à partir du $15^{\mathrm{e}}$ siècle à proximité d'un grand bloc de cette même grappe et un dépôt de trois monnaies romaines des premières décennies du premier siècle ap. J.-C à proximité d'un affleurement rocheux situé $25 \mathrm{~m}$ à l'aval (ouest) du noyau principal central. Un clou de chaussure romain, qui pourrait être augustéen ${ }^{53}$, a enfin été retrouvé sur

52. Ces billes d'un poids de $12 \mathrm{~g}$ ne sont pas encore identifiées précisément et pourraient correspondre à des sous-munitions d'obus Shrapnel.

53. PTC18-024 d'après Volken et al. (2011 : 360). Dans l'attente d'études comparables, les datations proposées dans le cadre de cette recherche pionnière sont à considérer avec réserve. 
le Col des Becs Noirs situé 450 m à l'estnord-est du noyau principal nord à 2667 m d'altitude.

En l'absence de mobilier dans une couche bien stratifiée et associée à une cabane ou à un abri, il est impossible de dater avec certitude, en relatif comme en absolu, les noyaux d'occupation du Plan de Tcholeire. La datation obtenue par le radiocarbone sur le local 16 du noyau central permet de placer l'occupation de ce dernier au plus tôt vers le milieu du $4^{\mathrm{e}}$ siècle av. J.-C. ${ }^{54}$ Cela dit, un potentiel "effet vieux-bois ", de par la réalisation de la mesure sur un charbon de bois, peut être envisagé. Il est donc plausible que la fourchette chronologique dans laquelle s'intègrent les occupations du Plan de Tcholeire soit similaire à celle des autres sites explorés dans la région. Toutefois, les plus de cinquante abris et fonds de cabanes questionnent quant au nombre de personnes ayant occupé la position. En contexte de montagne, l'économie de moyens implique plutôt une réutilisation des structures existantes que de nouvelles constructions. Il semble ainsi que seule la présence de plus d'une centaine de personnes à un moment donné puisse expliquer autant d'aménagements. Avec beaucoup moins d'individus, les abris déjà construits auraient probablement suffit et auraient été réutilisés sans nouveaux ajouts. À la périphérie des aménagements, les dépôts ou pertes de monnaies et les autres objets isolés pourraient n'être que les indices de réutilisations ponctuelles ou de passages postérieurs et ne permettent pas de dater l'ensemble des structures.

Les abris et fonds de cabanes du Plan de Tcholeire possèdent ainsi plusieurs ca- ractéristiques similaires aux abris et fonds de cabanes frustes des moraines à la périphérie du Mur (dit) d'Hannibal et pourraient participer d'un phénomène similaire. On y retrouve une quasi-absence de mobilier ou de niveaux d'occupation, des datations incertaines et un questionnement quant aux moyens investis par rapport à l'effectif des occupants. Le nombre approximatif de structures est proche. Les aménagements sont frustes et les espaces de petites dimensions. L'ensemble de ces critères semble pouvoir être mis en relation avec la présence de nombreux individus mais durant une période courte à très courte, ce qui expliquerait la quantité de structures mais également leur faible qualité et l'absence de dépôts ou de pertes de matériel.

L'hypothèse que nous formulons pour ce site est qu'il aurait servi ponctuellement de zone de bivouac ou de campement de marche pour une ou plusieurs troupes lors de déplacements qui pourraient être associés aux positions de l'arête de Barasson. L'absence de clous de chaussures dans et à proximité directe des bâtiments pourrait signifier la présence de troupes sans caligae ou un stationnement de très courte durée n'ayant pas occasionné de pertes. Sans éléments permettant de poser une chronologie entre les structures ou entre les noyaux et les grappes, il reste cependant tout à fait envisageable que l'utilisation de tous les aménagements ne soit pas synchrone. La présence de mobilier romain postérieur à l'époque tardo-républicaine illustre, de la même manière qu'au Mur (dit) d'Hannibal, des passages sur la position qui seraient postérieurs à sa probable occupation tardo-républicaine.

54. BE-11650.1.1, niveau d'occupation UT009, charbon de bois (essence non-déterminée), 361 (95.4\%) 196calBC; OxCal v4.3.2 (Bronk Ramsey, 2017), r5, IntCal13 atmospheric curve (Reimer et al., 2013). 


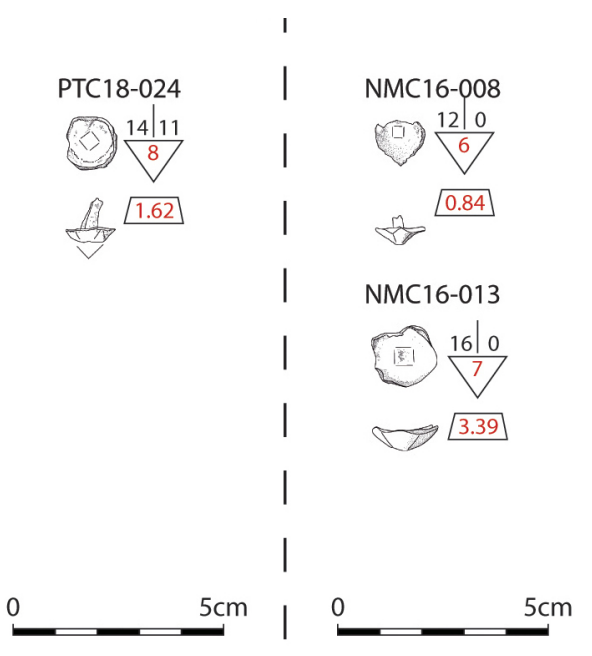

Figure 10. Plan de Tcholeire et Mont Carré, Planche présentant des sélections du mobilier métallique récolté sur les sites ou à leurs périphéries directes, P.-E. Mottiez et R. Andenmatten (RAMHA 2020), Plan de Tcholeire : PTC18-024, fer, clou de chaussure et Mont Carré : NMC16-008, NMC16013, fer, clous de chaussures.

Seules des investigations complémentaires pourraient permettre d'obtenir de nouveaux moyens de datation sur ce site et ainsi en améliorer la compréhension.

\subsection{Le Bonhomme du Tsapi (Liddes et Bourg-Saint-Pierre, Valais, Suisse, $2802 \mathrm{~m}$ )}

Le site archéologique du sommet du Bonhomme du Tsapi, à $2802 \mathrm{~m}$, a été repéré par un membre du groupe de recherches durant l'automne 2019. Il n'avait jamais été mentionné jusqu'ici et n'a pas encore fait l'objet d'investigations. Seul un premier repérage avec un relevé photographique a été réalisé.

Surplombant le Val d'Entremont, le Bonhomme du Tsapi est l'extrémité d'une arête rocheuse du massif des Combins au-dessus de Bourg-Saint-Pierre. Une sorte de plateau irrégulier constitué de cordons morainiques et de zones d'éboulis sur près de $6000 \mathrm{~m}^{2}$ se situe au sommet de cette montagne. Le lieu possède un extraordinaire point de vue. Avec son unique accès aisé par une arête, il est naturellement protégé sur tous ses côtés par des pentes abruptes et offre des conditions très favorables à l'installation en regard de son altitude.

Une quinzaine de fonds de cabanes y a été repérée et la position devrait faire l'objet d'investigations préliminaires en 2021. Les caractéristiques des petits bâtiments, leur organisation ainsi que la situation de la position rendent plausible une occupation contemporaine ou tout au moins se rapprochant fonctionnellement de celle du Mur (dit) d'Hannibal. Ce dernier n'est en effet situé qu'à $2.5 \mathrm{~km}$ au nord et un lien visuel direct relie les deux sites.

\subsection{Le Mont Carré (Nendaz, Valais, Suisse, $2385 \mathrm{~m}$ )}

À l'aval d'un col reliant le Val de Nendaz et le Val d'Hérens, le site archéologique du Mont Carré est positionné sur un plateau à la limite entre des dépôts morainiques quaternaires et des affleurements de roche-mère. Situé à env. 2385 m d'altitude, il s'agit du site le plus bas de notre corpus. On doit également relever sa position très éloignée des autres sites valaisans, tous documentés dans le Val d'Entremont. Cependant le lieu bénéficie d'un contexte proche des sites d'Entremont : à proximité d'une voie de passage, avec de grandes distances de visibilité, en un lieu très probablement isolé des habitats permanents. La position a fait l'objet de deux 


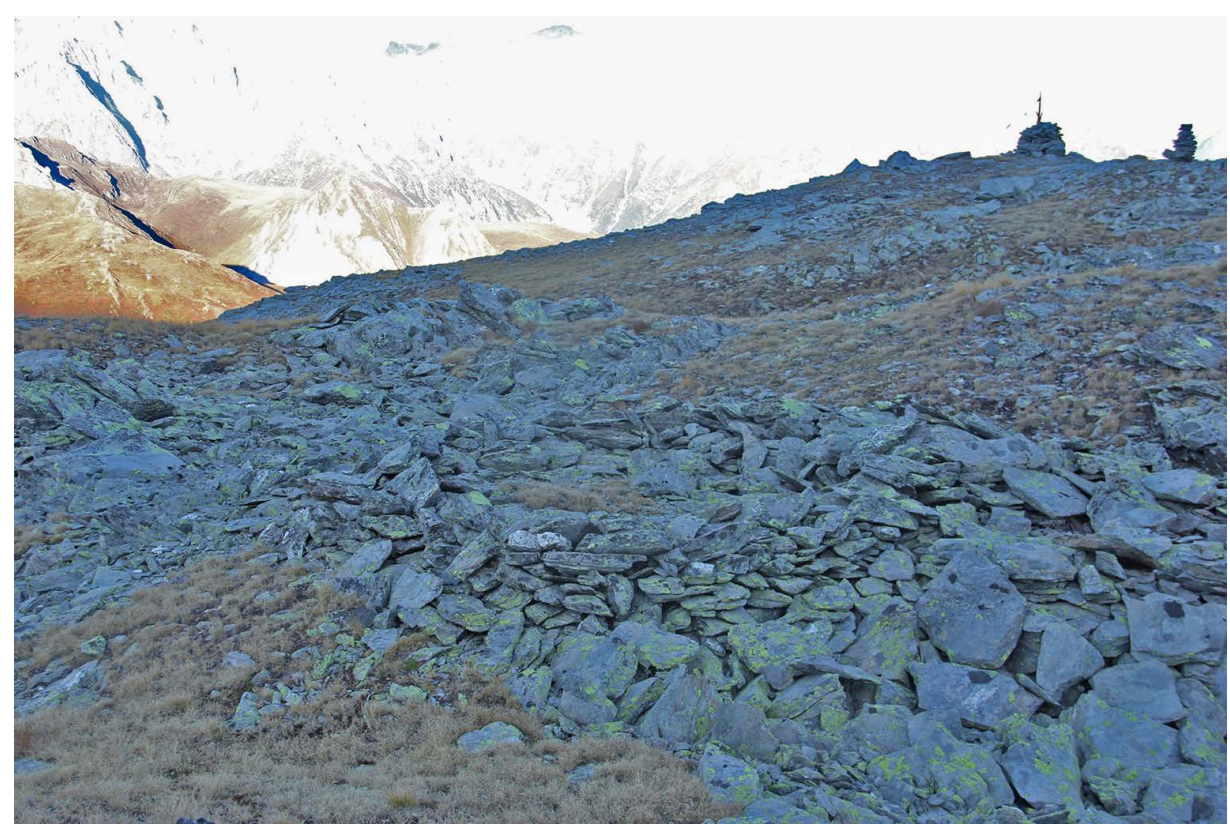

Figure 11. Bonhomme du Tsapi, cabane photographiée lors du premier repérage (CRAMHA 2019).

journées de documentation en 2016 et d'une journée d'investigation en 2018 avec une équipe de 6 personnes en moyenne. Des prospections ciblées, quelques petits sondages et un relevé général du site ont été réalisés. ${ }^{55}$

Les trente fonds de cabanes repérés présentent des caractéristiques similaires. Un abri, constitué d'un muret en forme de fer à cheval qui semble beaucoup plus récent que les autres structures, fait cependant exception. Plusieurs des sondages réalisés dans les fonds de bâtiments ont livré des charbons de bois en couche et l'un a même permis de fouiller une partie d'un petit foyer. Trois périodes d'occupation du site ont ainsi pu être définies à partir des datations par le radiocarbone. Les deux phases anciennes, au Néolithique moyen ${ }^{56}$ et à l'âge du Bronze final, ${ }^{57}$ sont

55. Andenmatten et Pignolet (2016b : 232) ; Andenmatten et al. (2020, à paraître).

56. BE-11646.1.1, colluvions UT017, charbon de bois (essence non-déterminée), 4332 (85.1\%) 4227calBC / 4201 (9.0\%) 4169calBC / 4091 (1.3\%) 4080calBC et BE-11929.1.1, colluvions UT017, charbon de bois (essence non-déterminée), 4323 (11.8\%) 4290calBC / 4267 (33.2\%) 4222calBC / 4208 (30.4\%) 4155calBC / 4131 (20.0\%) 4066calBC; OxCal v4.3.2 (Bronk Ramsey, 2017), r5, IntCal13 atmospheric curve (Reimer et al., 2013).

57. BE-11643.1.1, niveau d'occupation UT004, charbon de bois (essence non-déterminée), 1018 (95.4\%) 907calBC et BE-11928.1.1, niveau d'occupation UT004, charbon de bois (essence non-déterminée), 1026 (95.4\%) 914 calBC; OxCal v4.3.2 (Bronk Ramsey, 2017), r5, IntCal13 atmospheric curve (Reimer et al., 2013). 


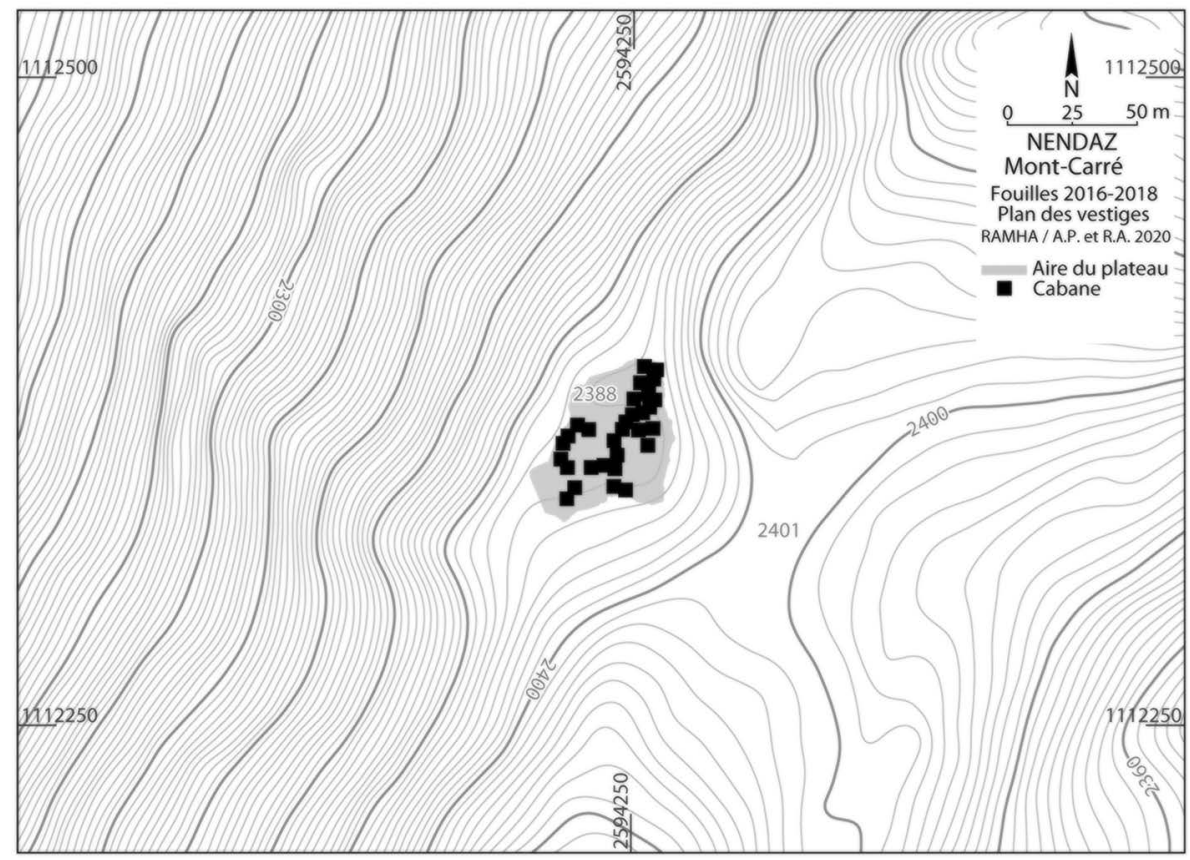

Figure 12. Mont Carré, Plan des vestiges, (C) RAMHA 2020.

attestées uniquement par des charbons de bois récoltés dans des niveaux de colluvions ou d'occupation sans liens stratigraphiques assurés avec l'aménagement et l'utilisation de fonds de cabanes.

La troisième et principale phase d'occupation est illustrée par des datations sur des charbons de bois issus de niveaux de défournement et d'un foyer dans trois locaux différents. Les trois analyses par le radiocarbone donnent une fourchette comprise entre le premier quart du quatrième siècle et le milieu du premier siècle av. J.-C. ${ }^{58}$ Les datations ayant été réalisées sur des fragments de charbon de bois, on peut envisager qu'elles soient légèrement ancienne de par un «effet vieux bois» tandis que deux clous de chaussures romains ${ }^{59}$ découverts à la périphérie des bâtiments, et une demi-monnaie, ${ }^{60}$ retrouvée dans un niveau d'occupation à l'intérieur d'une cabane, pourraient per-

58. BE-11642.1.1, niveau d'utilisation de foyer UT002, charbon de bois (pinus type cembro), 361 (95.4\%) 201calBC ; BE-11644.1.1, niveau d'occupation UT006, charbon de bois (picea / larix), 386 (26.1\%) 350calBC / 311 (69.3\%) 209calBC et BE-11645.1.1, niveau d'occupation UT011, charbon de bois (essence non-déterminée), 203 (89.7\%) 87calBC / 79 (5.7\%) 56calBC; OxCal v4.3.2 (Bronk Ramsey 2017), r5, IntCal13 atmospheric curve (Reimer et al., 2013).

59. Les clous NMC16-008 et NMC16-013 sont présentés en fig. 10. Le second clou, par ses grandes dimensions, permet d'envisager le passage d'une personne équipée de caligae, avant ou en 16/15 avant J.-C.

60. Ce demi-as (NMC16-012) est très mal conservé et son émission tout comme sa datation restent discutées. 
mettre de rattacher, du moins ponctuellement, l'occupation de cette position à une fourchette chronologique de peu postérieure ou similaire à la majorité des autres sites présentés dans cette contribution. Il reste cependant difficile de comprendre la fonction de cette occupation qui semble combiner une position ouverte avec des bâtiments similaires à ceux de la zone protégée d'un site tel que le Mur (dit) d'Hannibal. La position du Mont Tantané en Vallée d'Aoste présente des caractéristiques proches et pourrait être un parallèle intéressant. Cependant, que ce site puisse relever d'une autre problématique doit également être envisagé. Avec ses phases d'occupation successives, il semble dans tous les cas de grand intérêt d'envisager à moyen terme des investigations plus poussées sur cette position.

\subsection{Les sites valdôtains}

Comme évoqué ci-dessus, ces lieux feront l'objet d'une autre publication. On peut cependant relever la présence d'une quinzaine de sites présentant des caractéristiques similaires aux positions valaisannes sur le territoire de la Région autonome Vallée d'Aoste. Parmi ces positions, le site du Mont Tantané, étudié durant plusieurs campagnes successives, fait office d'exception. ${ }^{61}$ Deux sites supplémentaires, la Punta Fetita et la Tour Ronde ont fait l'objet d'investigations préliminaires communes en 2018 et 2019 (levé d'un plan général, prospections au détecteur de métaux et sondages ciblés). ${ }^{62}$ Les résultats obtenus de l'exploration de ces trois emplacements, dont seule la Punta Fetita est fortifiée, ${ }^{63}$ permettent d'assurer une fourchette d'occupation principale de ces sites similaire à celle du Mur (dit) d'Hannibal. Des militaria romains sont de plus présents sur les trois positions.

\section{Discussions et perspectives}

\subsection{Archéologie et Histoire}

Six des sept sites présentés dans cette contributions possèdent donc des caractéristiques communes et certaines de leurs occupations s'insèrent dans une fourchette de datation similaire qui recoupe les deux derniers tiers du premier siècle avant J.-C., entre l'époque césarienne et l'époque augustéenne précoce. L'analyse combinée des positions valaisannes et valdôtaines amène à envisager une association de ces sites avec une série d'opérations militaires romaines menées entre 35 et 25 av. J.-C. autour et en Vallée d'Aoste, territoire du peuple indigène des Salassi. ${ }^{64}$ Une synthèse des sources archéologiques et historiques mettant en perspective les découvertes valaisannes a également permis de discuter et d'envisager l'entrée des populations du Valais central (Seduni et Veragri) et du Chablais (Nantuates) dans l'imperium Romanum 10 à 20 ans avant les dates communément retenues de 25 ou 15 avant J.-C. ${ }^{65}$

Les modalités de la mise en place de l'hégémonie romaine dans les Alpes suisses ont aussi été revisitées dans le

61. Armirotti (2003 : 140-141) ; Mezzena (2004: 157); Bertocco (2017: 87 et 90).

62. Surintendance pour les activités et les biens culturels et groupe scientifique RAMHA.

63. Andenmatten et al. (2020, à paraître).

64. Andenmatten et Aberson (2019: 223-226); Aberson et Andenmatten (2021, à paraître).

65. Andenmatten et Aberson (2019: 226) ; Aberson et Andenmatten (2021, à paraître). 
cadre de recherches menées par Stefanie Martin-Kilcher ces dix dernières années ${ }^{66}$; recherches dont notre travail bénéficie largement. Il semble ainsi qu'en plusieurs cas, une présence romaine plus précoce qu'attendue puisse être archéologiquement attestée dans les Alpes et qu'il faille libérer les interprétations archéologiques du magnétisme des sources historiques. ${ }^{67}$ Dans les Alpes orientales de la Suisse, les découvertes militaires romaines précoces étaient traditionnellement associées à la "Conquête des Alpes " lors de l'opération combinée menée par Drusus et Tibère en 16/15 av. J.-C. Des arguments archéologiques permettent cependant aujourd'hui d'envisager une présence romaine dans la région près d'une quinzaine d'années auparavant. ${ }^{68}$

\subsection{Questions de datation}

Des éléments négligés jusqu'ici, tels que les clous de chaussures, prennent une importance significative dans la définition de la chronologie des sites de cette période. En effet, des clous de chaussures apparaissent sur les sites occupés par l'armée romaine probablement entre le deuxième quart et le deuxième tiers du $1^{\text {er }}$ siècle avant J.-C. Ces clous possèdent généralement un diamètre supérieur à 15 mm jusqu'en 16/15 avant J.-C. et les clous de cette taille semblent ensuite disparaître dans les sites datés postérieurement à 15 avant J.-C. Ces éléments constitueraient ainsi d'intéressants termini post et ante quem. ${ }^{69}$
Concernant les datations par le radiocarbone, malgré une sélection des charbons de bois par l'anthracologue, des « effets vieux-bois " récurrents de 100 à 300 ans ont été observés sur les charbons de bois en regard du mobilier et des datations par le radiocarbone sur les macrorestes alimentaires ; ceci même en l'absence de bois architectural. Cette observation appelle à la prudence quant à la surinterprétation des datations par le radiocarbone qui ne peuvent dans le contexte de ces sites qu'être considérées comme des termini post quem. Bien que ces sites soient relativement pauvres en objets, les ensembles de mobilier retrouvés en stratigraphie dans les bâtiments y constituent un intéressant corpus qui pourrait servir de référence pour la période à moyen terme.

Les datations des occupations principales de la majorité des positions explorées se recoupent mais, les utilisations des sites étant très probablement événementielles, une synchronie reste impossible à prouver par l'archéologie ou le radiocarbone. L'établissement d'une courbe dendrochronologique relative intégrant les bois glaciaires et des charbons de bois est en cours d'expérimentation pour tenter $d^{\prime} y$ remédier. ${ }^{70}$

\subsection{Quel dispositif et dans quel cadre?}

Une tentative de catégorisation des positions explorées a été entreprise et, bien qu'imparfaite, elle permet de mettre en évidence les similitudes et les différences entre les positions. ${ }^{71}$ Les sites les plus dé-

66. Martin-Kilcher (2011) ; Martin-Kilcher (2015) ; Martin-Kilcher (2016) ; Martin-Kilcher (2021, à paraittre).

67. Aberson et al. (2019: 21-22).

68. Martin-Kilcher (2011 : 43) ; Roth-Rubi et al. (2004 : 48-49).

69. Martin-Kilcher (2021, à paraître).

70. Projet avec Fabien Langenegger du Laboratoire de dendrochronologie de Neuchâtel.

71. Andenmatten et Aberson (2019: 220). 
Autour du Mur (dit) d'Hannibal : appréhender un " dispositif militaire » du premier siècle av. J.-C. dans les

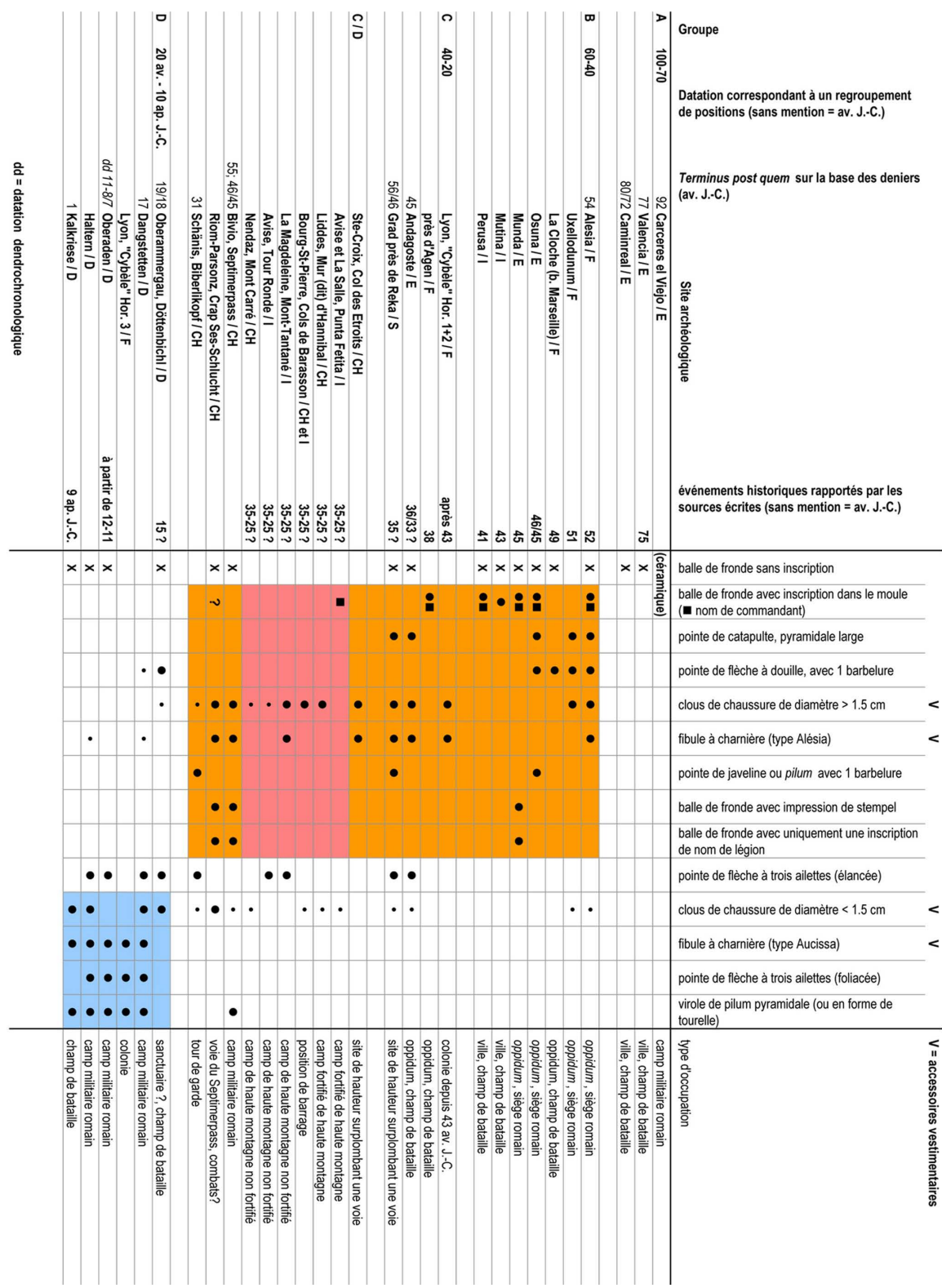

Figure 13. Tableau de " combinaison des armes et des accessoires vestimentaires caractéristiques des sites archéologiques datés et de comparaison avec les sites de la région des Alpes centrales ; avec les termini post quem des deniers ", d'après Martin-Kilcher (2011: 54) et Martin-Kilcher (2015: 244). Les sites en cours d'étude dans les Alpes poenines ainsi que le site de Döttenbichl, d'après Zanier (2016), ont été intégrés dans le cadre de cet article (@ RAMHA 2020). 
veloppés sont les enceintes fortifiées telles que le Mur (dit) d'Hannibal. Ces premières ont quelques bâtiments d'habitation dont les caractéristiques sont proches des occupations de fond de vallée, de nombreux fonds de cabanes et peuvent, ou non, posséder des aménagements défensifs ainsi que des structures de campement périphériques. La deuxième catégorie correspond aux positions préparées avec fortification de barrage sur des points de passage mais présentant des structures visibles d'habitat frustes et en petit nombre, tels les Cols de Barasson ou d'Annibal. Un troisième type comprendrait les habitats non-fortifiés avec des cabanes similaires aux sites avec enceinte, tels le Mont Carré. Et une dernière catégorie engloberait des sites de campement très événementiels avec uniquement des aménagements frustes comme le site du Plan de Tcholeire. La variété des positions n'est pas encore expliquée et pourrait résulter d'occupants, de missions ou de datations légèrement différentes. Les altitudes ainsi que les situations " tactiques » des sites ont également été envisagées comme des critères. Toutes ces variantes ont fait et font l'objet de réflexions ${ }^{72}$ mais seule l'exploration de positions supplémentaires permettra d'améliorer notre compréhension de l'ensemble du phénomène.

Les datations actuellement à disposition restent en effet encore trop larges pour tenter une association de plusieurs sites à un même événement connu ou inconnu. La majorité des positions que nous avons explorées pourraient cependant avoir été occupées ou parcourues par des troupes romaines entre 35 et 25 av. J.-C., durant une période d'instabilité et de conflits quasi permanents entre Rome et les Salassi. En effet, ces événements sont les seuls à donner une explication plausible à un tel déploiement en haute montagne. ${ }^{73}$ Le blocus mis en place par Gaius Antistius Vetus en 35-34 avant J.-C. autour du territoire salasse est l'un des événements envisagés mais les opérations de $M$. Valerius Messala Corvinus entre 30 et 28-27 avant J.-C. ou d'Aulus Terrentius Varro Murena en 25 avant J.-C., dont nous ignorons les détails tactiques, pourraient également avoir nécessité de tels aménagements. Une répartition entre plusieurs de ces événements ou l'attribution de certains sites à des événements non relatés dans les sources restent également possibles.

Cependant, l'inter-visibilité entre plusieurs des sites étudiés doit être relevée. Des contacts intersites étaient ainsi possibles mais également d'éventuelles communications à longue distance par plusieurs positions. Les sites de la région du Grand-Saint-Bernard auraient ainsi eu la possibilité de transmettre un message simple jusqu'aux positions proches du Petit-Saint-Bernard, à env. 30 km, en seulement deux intermédiaires.

72. Andenmatten et Aberson (2019: 220).

73. L'occupation de telles positions offrirait de nombreux avantages tactiques : occuper des positions favorables du relief qui permettent non seulement la récolte d'informations mais aussi la conduite d'opérations dans plusieurs directions ; contrôler des passages obligés d'altitude pour en interdire l'utilisation à l'adversaire et s'assurer la liberté de déplacement de ses propres troupes ; créer un dispositif d'encerclement ou de fractionnement des unités de la partie adverse pour en limiter les capacités d'approvisionnement et de soutien mutuel ; assurer des communications à longue distance dans un environnement compartimenté (intervisibilité) ; stationner une partie de ses forces à distance des lieux les plus peuplés. Andenmatten et Aberson (2019: 221-223). 
Il est donc plausible de considérer les positions présentées ci-dessus, à l'exception du site du Mont Carré qui semble pour l'instant particulier, comme faisant partie d'un dispositif occupé par des troupes romaines durant les décennies agitées ayant mené à l'intégration des Alpes poenines dans l'imperium Romanum.

\subsection{Perspectives de collaborations et poursuites des recherches}

À côté des aspects historiques, l'étude de ces positions permet également d'explorer les modes d'opération et d'approvisionnement ainsi que la vie quotidienne et l'organisation de troupes romaines de la période tardo-républicaine en montagne. L'environnement dictant une grande partie des choix tactiques lors d'opérations militaires, des comparaisons avec des vestiges de la même période dans d'autres régions montagneuses pourraient se révéler d'un grand intérêt. On peut aussi rappeler que certains officiers romains, tel Gaius Antistius Vetus, ont servi aussi bien dans les Alpes poenines que dans les montagnes de Cantabrie, ${ }^{74}$ et ont probablement développé des tactiques adaptées à ces milieux.

Une monographie sur les recherches autour du Mur (dit) d'Hannibal est planifiée dans les deux ans à venir et sera l'occasion de proposer une synthèse des questions abordées dans cet article. Espérons ainsi que la présente contribution permettra de renforcer les échanges entre des groupes de chercheurs s'intéressant aux mêmes problématiques dans des régions de montagne géographiquement éloignées mais plus proches qu'il n’y paraît.

\section{Références bibliographiques}

Abréviations :

$A A S=$ Annuaire d'Archéologie Suisse

$A S S P A=$ Annuaire de la Société Suisse de Préhistoire et d'Archéologie

Bulletin AFEAF = Bulletin de l'Association française pour l'étude de l'âge du Fer

$B E P A A=$ Bulletin d'Etudes Préhistoriques et Archéologiques Alpines

$B S B A C=$ Bollettino della Soprintendenza per i beni e le attività culturali

$J R A=$ Journal of Roman Archaeology

$N A B=$ Notizie Archeologiche Bergomensi

74. Florus, Abrégé d'histoire romaine, 4, 12. 
Aberson, M. ; Andenmatten, R. (2021). «L'entrée du Valais dans l'imperium Romanum : indices archéologiques et témoignages historiques". À : Gregori, G. L. ; Dell'ErA, R. (éds.). Les Romains dans les Alpes. Histoire, archéologie, épigraphie. Atti del Convegno internazionale, Losanna, 13-15 maggio 2019, à paraître.

Aberson, M. ; Andenmatten, R. ; Casini, S. ; Fossati, A. E. ; Wachter, R. (2021). « Entre Celtes et Romains : la dédicace à Poeninos du Mur (dit) d'Hannibal ». À : Dupraz, E. ; Estarán Tolosa, M. J. ; Aberson, M. (éds.) : Des mots pour les dieux. Dédicaces cultuelles dans les langues indigènes de la Méditerranée occidentale (Études Genevoises sur l'Antiquité 8). Bern, 309-332.

Aberson, M. ; Curdy, P. ; Ripart, L. (2019). "Territoires, frontières et peuples dans les Alpes : quelques réflexions méthodologiques ". BEPAA, 29-30, 13-38.

Andenmatten, R. (2011). Le Mur (dit) d'Hannibal: un site de haute montagne hors normes. Mémoire de Maîtrise de l'Université de Lausanne, non publié. Lausanne.

Andenmatten, R. ; Aberson, M. (2019). « Passer, occuper ou contrôler les Alpes poenines à la transition entre l'âge du Fer et l'époque romaine ». BEPAA, 29-30, 219-227.

Andenmatten, R. ; Armirotti, A. ; Pignolet, A. (2020). " Projet de recherches autour du Mur (dit) d'Hannibal (RAMHA)». À : BRunetTi, C. (dir.). Chronique des découvertes archéologiques dans le canton du Valais en 2018. Vallesia, 73, pp. 3, 19, 26, 31.

Andenmatten, R. ; Paccolat, O. (2010). «Liddes, district d'Entremont, Creux de Boveire, 'Mur (dit) d'Hannibal' ». À : Wiblé, F. (dir.). Chronique des découvertes archéologiques dans le canton du Valais en 2009 et 2010. Vallesia, 65, 311-313.

Andenmatten, R. ; Paccolat, O., avec les contributions de Mermod, O. ; Schlumbaum, A. et Studer, J. (2012). «Le Mur (dit) d'Hannibal : un site de haute montagne de la fin de l'âge du Fer ». $A A S, 95,77-95$.

Andenmatten, R. ; Pignolet A. (2015a). «Liddes, district d'Entremont, Creux de Boveire, 'Mur (dit) d'Hannibal'». À : Wiblé, F. (dir.). Chronique des découvertes archéologiques dans le canton du Valais en 2014. Vallesia, 69, 525-527.

- (2015b). «Liddes, district d'Entremont, Creux de Boveire, 'Mur (dit) d'Hannibal' ». À : WIBLÉ, F. (dir.). Chronique des découvertes archéologiques dans le canton du Valais en 2015. Vallesia, 70, 297-299.

- (2016a). «Projet de recherches archéologiques sur le Mur (dit) d'Hannibal. Un établissement fortifié tardo-républicain de haute montagne ». BEPAA, 27, 313-316.

- (2016b). "Liddes, district d'Entremont, Creux de Boveire, 'Mur (dit) d'Hannibal' ; BourgSaint-Pierre, district d'Entremont, 'Col ouest de Barasson', 'Col est de Barasson' et 'Col d'Annibal' ; Nendaz, disctrict de Conthey, 'Mont Carré' ». À : Brunetri, C. (dir.), Chronique des découvertes archéologiques dans le canton du Valais en 2016. Vallesia, 71, 226-233 et 248.

- (2017). "Recherches archéologiques autour du Mur (dit) d'Hannibal à Liddes : Des sites fortifiés de haute montagne dans la région du Grand-Saint-Bernard (Suisse/Valais, Italie/Val d'Aoste) ». Bulletin AFEAF, 35, 41-44.

Armirotti, A. (2003). « Rete viaria e insediamenti minori nel territorio valdostano in epoca romana e tardoantica ". BEPAA, 14, 9-203.

Benedetti, S. ; Curdy, Ph. (2008). «Prospections au Col du Grand-Saint-Bernard : voies d'accès et passages latéraux ». À : Appolonia, L. ; Wiblé, F. ; Framarin, P. (dir), Alpis poenina, Grand-Saint-Bernard, une voie à travers l'Europe. Aoste : Projet Interreg III A Italie-Suisse 2000-2006, 377-390.

Bertocco, G. (2017). "Recenti scoperte protostoriche in Valle d'Aosta ». BEPAA, 28, 83-101.

Casini, S. ; Fossati, A.E. ; Motta, F. (2013). "L'inscrizione in alfabeto di Lugano al Mur d'Hannibal (Liddes, Valais) ». NAB, 21, 157-165.

Cibrario, G. (1901). "Mont Vélan ». Rivista mensile del Club Alpino Italiano, 10, 385-386.

DAUDrY, D. (2005). « Segnalazione e documentazione fotografica del villagio protostorico della Cime Noire ». BEPAA, 16, 157-175. 
Deyber, A. ; ZaAraoui, Y. ; Buffat, L. (2018). « Le Lampourdier. Un camp romain républicain témoin de la bataille d'Orange (6 octobre 105 av. n. è.) ". À : RedDÉ, M. (dir.). L'armée romaine en Gaule à l'époque républicaine. Nouveaux témoignages archéologiques. Glux-en-Glenne : Bibracte, Centre archéologique européen, 19-43.

Lattion, T. (1983). Réflexions à propos du "Mur d'Annibal». Archives privées, non publié. Liddes.

Martin-Kilcher, S. (2011). "Römer und gentes Alpinæ im Konflikt - archäologische und historische Zeugnisse des 1. Jahrhunderts v. Chr. ». A : Lehmann, G. A. ; Wiegels, R. (eds.). Fines imperii - imperium sine fine? Rahden : VML Vlg Marie Leidorf, 27-62.

- (2015). "Archäologische Spuren der römischen Okkupation zwischen Alpen und Hochrhein und die städtische Besiedlung der civitas Helvetiorum im 1. Jh. v. Chr. ». À : Moosbauer, G. ; Wiegels, R. (éds). Über die Alpen und über den Rhein ..., Beiträge zu den Anfängen und zum Verlauf der römischen Expansion nach Mitteleuropa. Berlin, Boston: De Gruyter, 235281.

- (2016). «Gentes Alpinae et Romains en conflit au I ${ }^{\text {er }}$ s. av. J.-C. Témoins archéologiques des campagnes des Alpes et impacts de l'occupation romaine entre les Alpes et le Rhin ». BEPAA, 27, 197-211.

- (2021). «La présence romaine dans les Alpes au premier siècle avant J.-C. L'apport des données archéologiques ". À : Gregori, G. L. ; Dell'Era, R. (éds.). Les Romains dans les Alpes. Histoire, archéologie, épigraphie. Atti del Convegno internazionale, Losanna, 13-15 maggio 2019, à paraître.

Mezzena, F. (2004). « Habitat protohistorique au Mont-Tantané ». BSBAC, 1, 157.

Pohanka, R. (1986). Die eisernen Agrargeräte der Römischen Kaiserzeit in Österreich. Oxford: Archaeopress.

Quartier-La-Tente, V. (2007). "L'énigme du Mur d'Hannibal ! Enfin une piste ». La vallée du Gd-St-Bernard, Liddes et Bourg-St-Pierre vous informent, 12-13.

Reboulaz, P. (2005). "Prima segnalazione di un antico villaggio, St-Barthelemy ». BEPAA, 15, 197-198.

Ribera i Lacomba, A. ; Calvo Galvez M. (1995). " La primera evidencia arqueológica de la destrucción de Valentia por Pompeyo ». JRA, 8, 19-40. <https://doi.org/10.1017/S1047759400015956>

Roth-Rubi, K. ; Schaltenbrand Obrecht, V. ; Schindler, P. M. (2004). « Neue Sicht auf die 'Walenseetürme' ». ASSPA, 87, 33-70.

SChiffer, M. B. (1986). "Radiocarbon dating and the "old wood" problem: The case of the Hohokam chronology ». Journal of Archaeological Science, 13 (1), 13-30. <https://doi.org/10.1016/0305-4403(86)90024-5>

Scholl, M.-J. (2015). Stabilisation des vestiges archéologiques en fer associés à des restes de bois : caractérisation du degré de minéralisation et refléxions sur leur comportement dans des bains alcalins. Mémoire de Maîtrise de la Haute Ecole Arc Conservation-Restauration de Neuchâtel, Berne et Jura, non publié. Neuchâtel.

Volken, M. ; Paccolat, O. ; Volken, S. (2011). «Les clous de chaussures du site de Pfyngut: les bases d'une typo-chronologie". À : Paccolat, O. (dir.), PFYN/FINGES, évolution d'un terroir de la plaine du Rhône, Le site archéologique de "Pfyngut" (Valais, Suisse). Lausanne : Cahiers d'archéologie romande, 315-387.

Zanier, W. (dir.) (2016). Der Spätlatène- und Frühkaiserzeitlische Opferplatz auf dem Döttenbichl südlich von Oberammergau. München : C.H. Beck. 